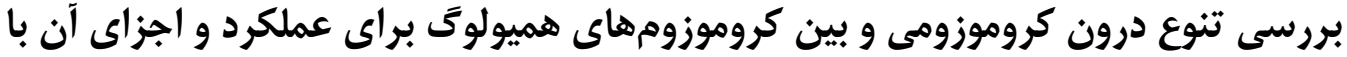

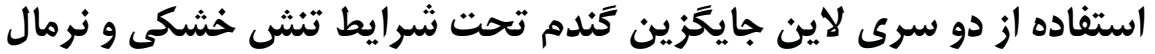

\author{
مهناز عليمحمدى'، شهرام محمدى ‘َ، بهروز شيران 'ّو محمد ربيعى
}

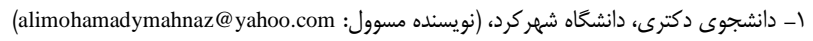

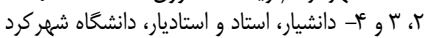

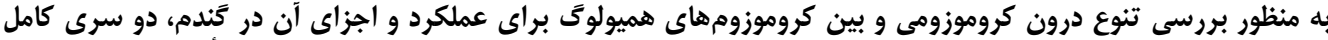

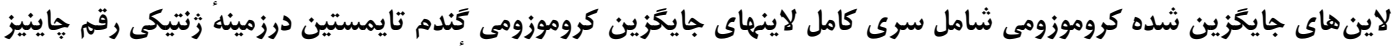

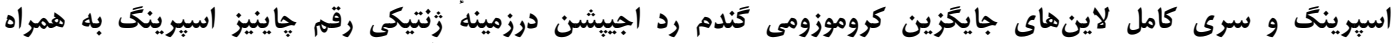

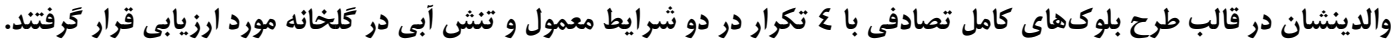

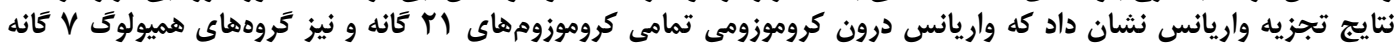

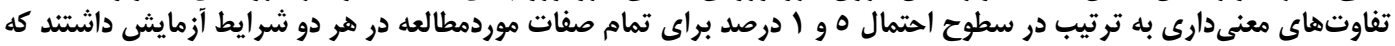

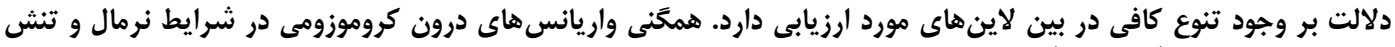

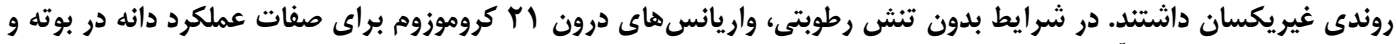

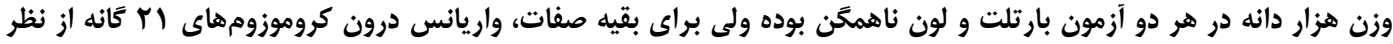

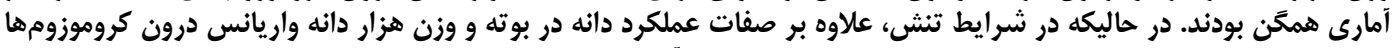

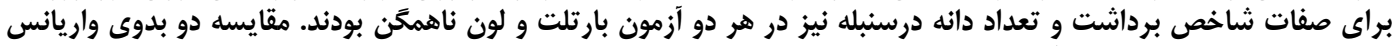

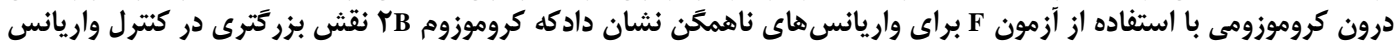

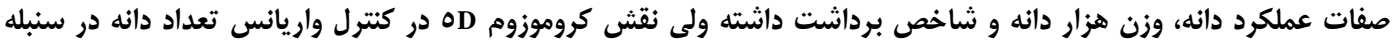

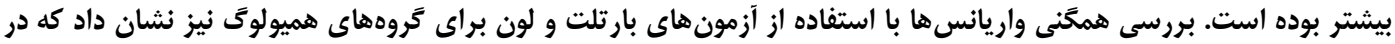

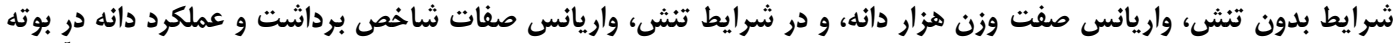

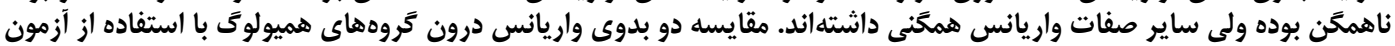

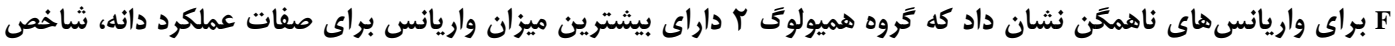

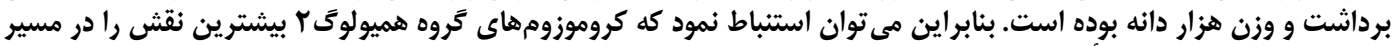

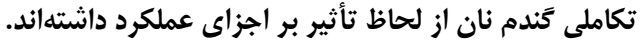

وازههاى كليدى: تنش خشكى، تنوع درون كروموزومى، تنوع بين كروموزومهاى هميولوَى، كندم، لاينهاى جايكزين كروموزومى

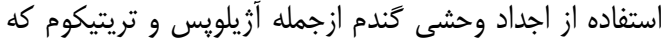

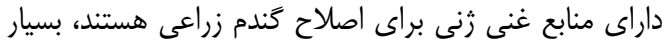

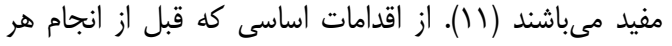

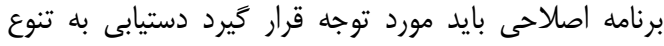

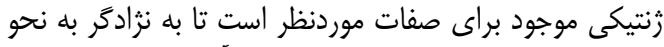

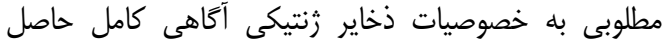

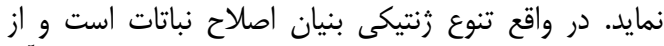

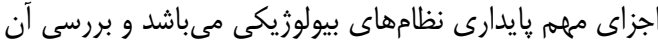

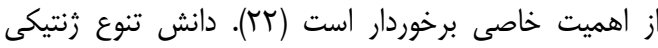

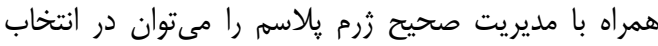

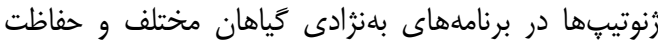

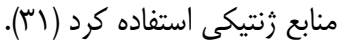

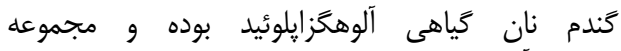

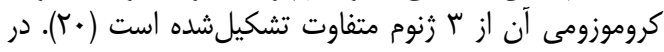

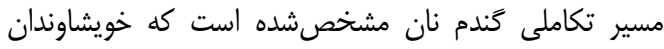

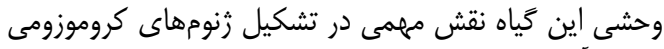

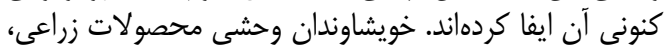

مقدمه

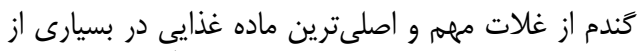

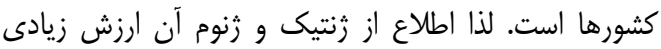

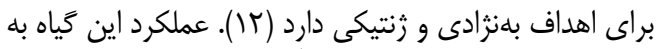

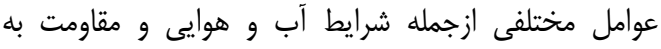

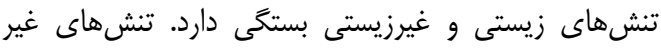

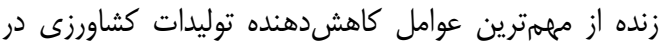

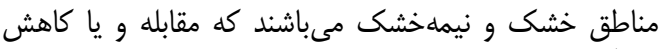

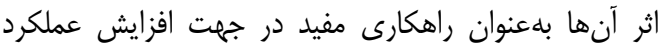

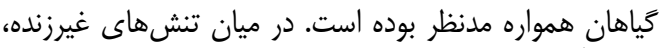

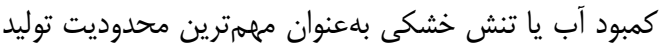

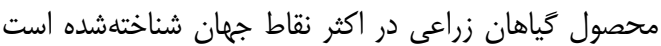

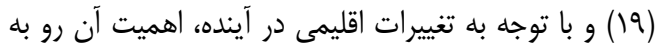

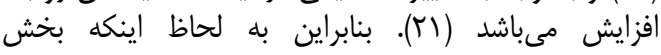

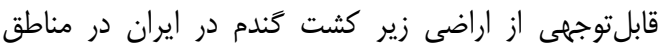

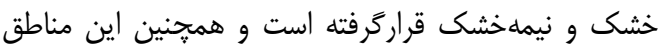
داراى دامنهاى از تنشهاى زيستى و غير زيستى مىباشند، 
كروموزومى Tim/Cs) و سرى كامل كاين لاينهاى جايخزين

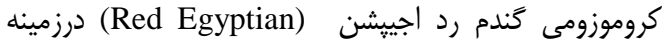

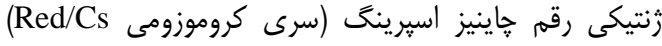

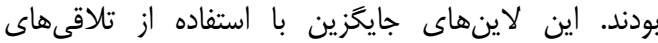

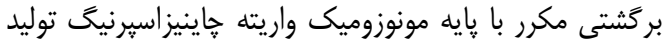
و تا نسل BC10 خالص كرديدهاند (

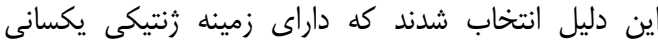

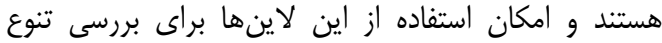

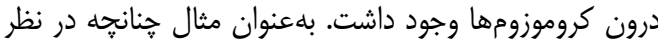

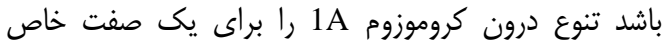

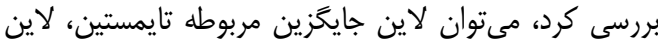
جايخزين مربوطه رد اجيشيشن و واريته

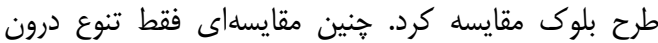

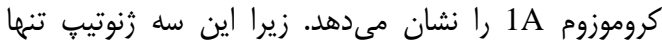

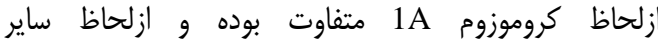
كروموزومها يكسان هستند.

در اين تحقيق تمامى مواد زنتيكى فوق (دو سرى لايكسين لاين

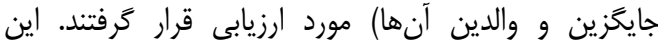

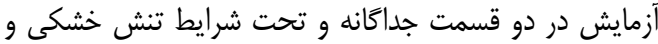

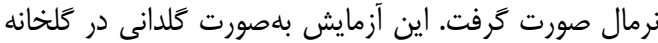

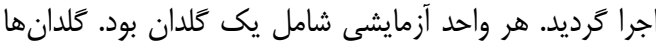

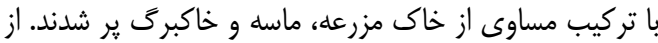

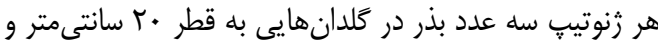

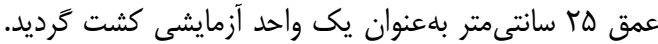

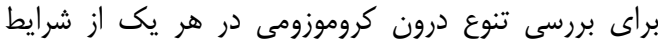

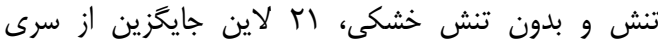

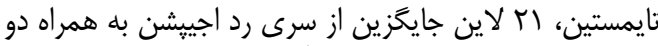

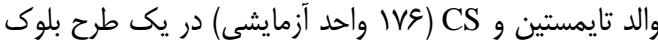

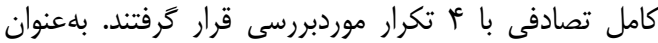

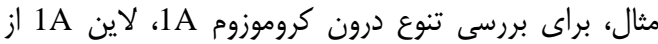

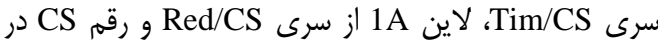

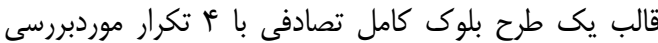

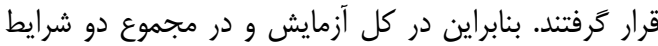

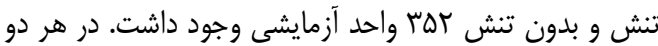

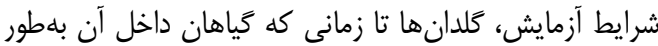

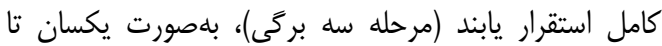

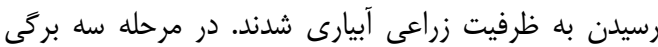

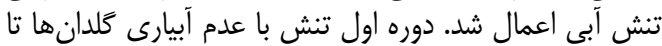

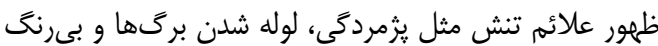

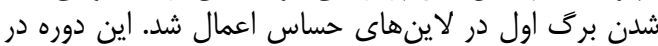

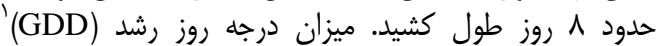

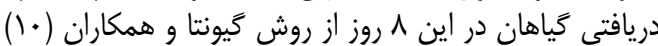

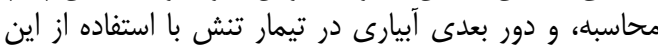

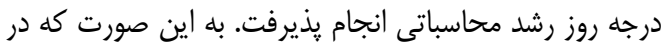

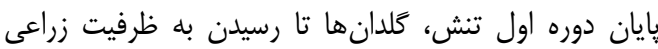

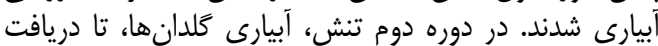

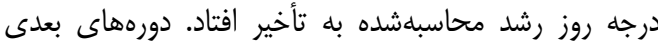

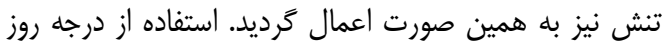
رشد سبب يكسانسازى شرايط تنش براى همين همه لاينهاى

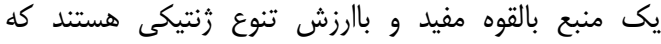

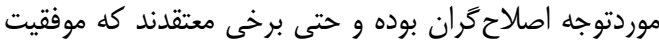

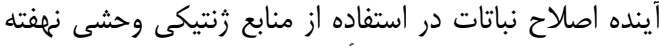

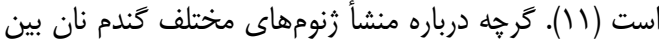

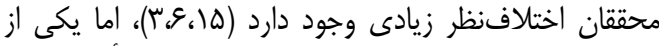

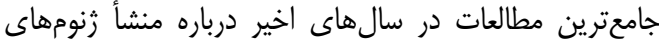

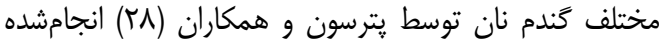

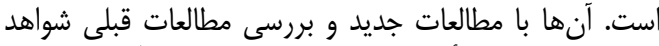

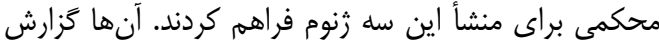

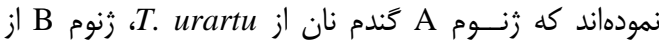
Ae. speltoides

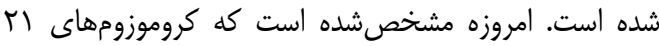

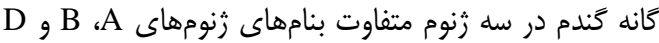

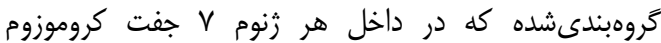

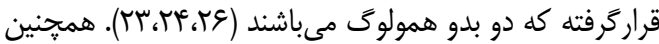

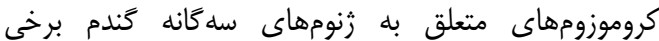

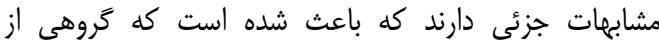
كروموزومها كه متعلق به زيه زنومهاى متفاوت

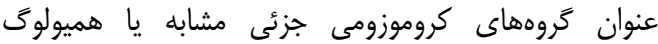

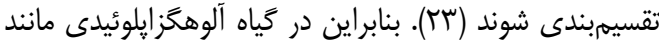

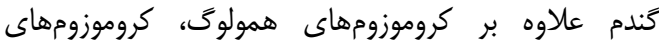
هميولوگ نيز وجود دارد.

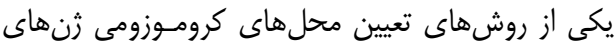

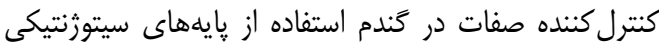

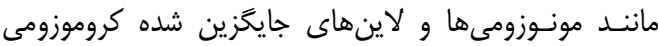

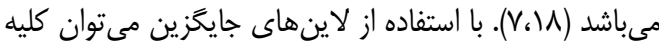

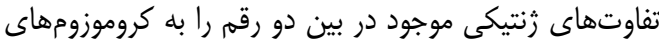

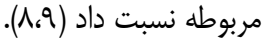

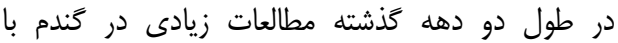

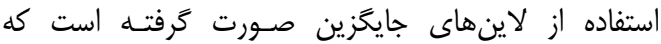

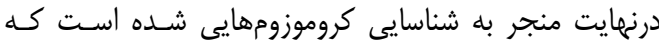

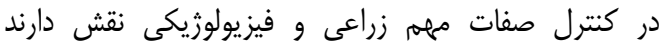

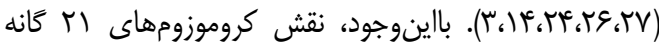

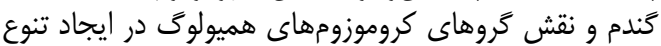

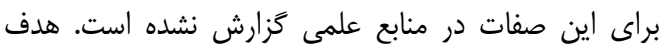

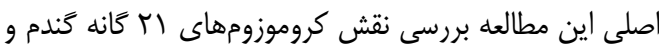

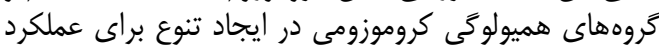

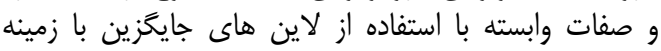

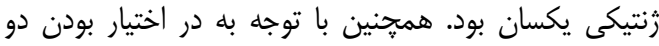

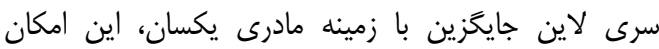

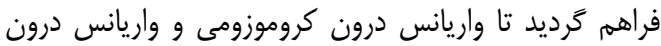

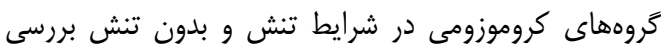
كردد.

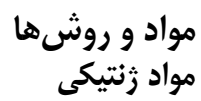

مواد زنتيكى اين مطالعه شامل سرى ستى كامل لاينهاى

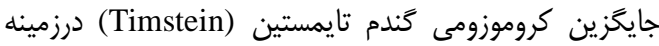

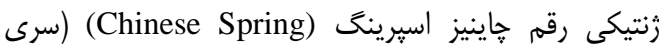




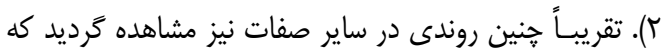

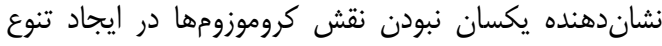

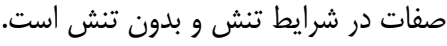

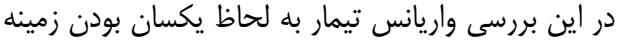

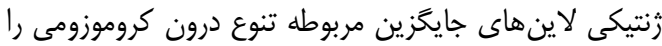

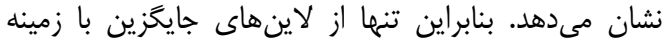

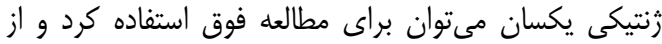

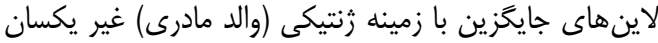

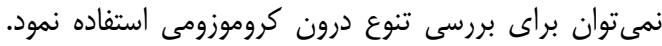

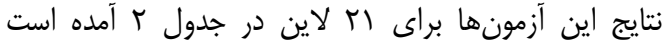

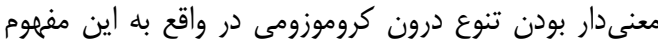

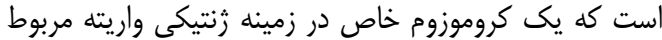

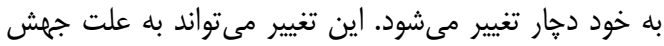

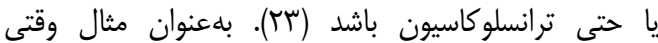

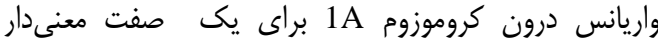

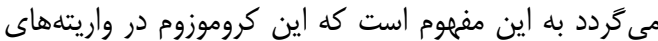

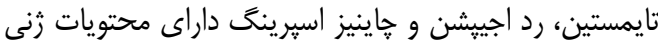

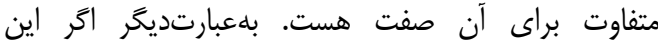
كروموزوم در هر سه واريته يكسان بان باشد، نبايد تنوع درون درون كروموزومى معنى دار كردمد درد.

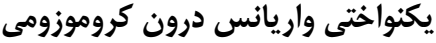

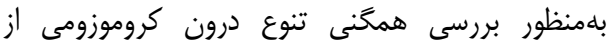

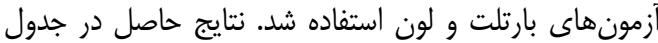

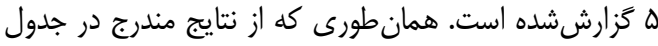

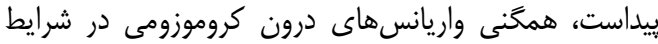
نرمال و تنش روندى غير يكسان داشته است. در شرايط بد بدون درون درون

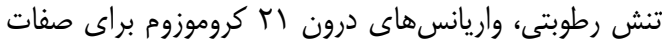

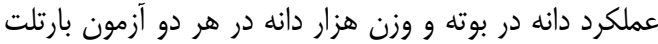

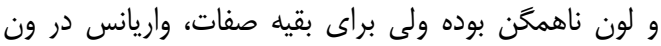

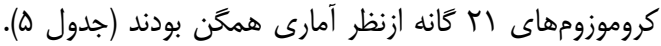

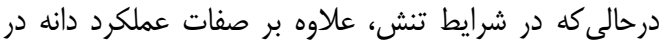

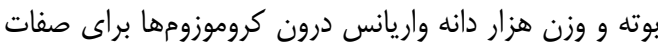

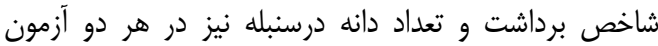

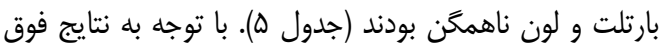

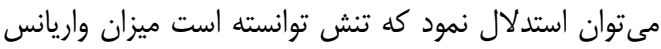

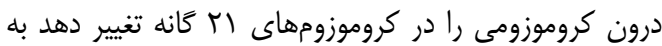

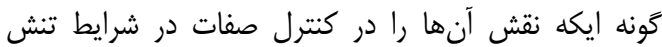

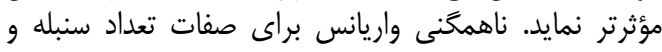

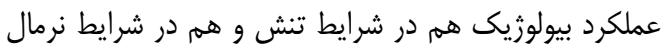

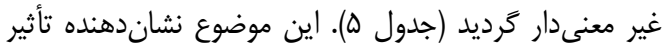

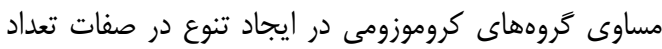

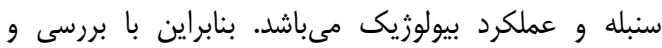

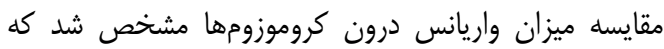

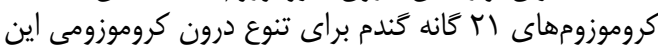

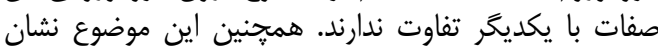

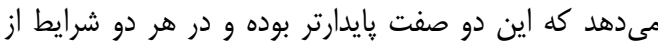

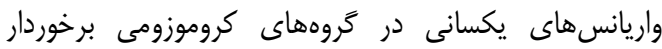

جايخزين و مستقل شدن تنش از نوسانات دمايى در طى مادى وزهاى مختلف در مراحل رشد كياه مى شودان

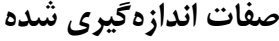

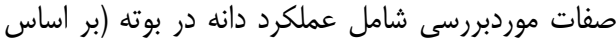

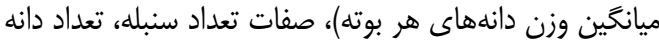

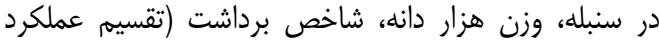

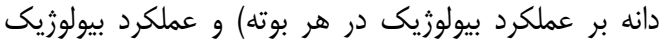

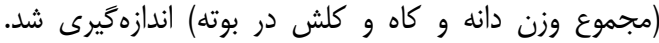

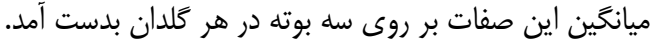

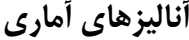

با توجه بله در اختيار بودن سرى إمهاى متفاوت لاينهاى

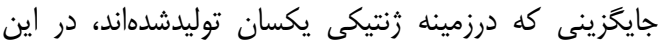

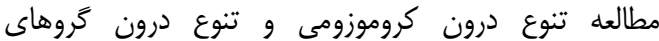

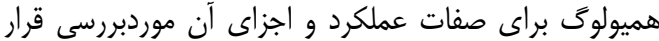

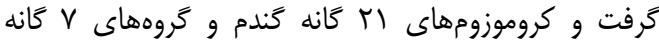

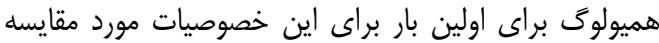

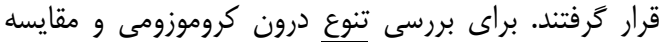

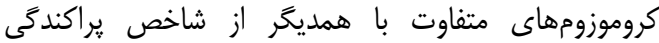

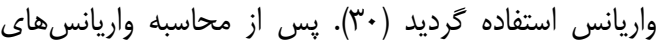

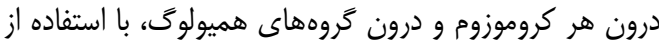

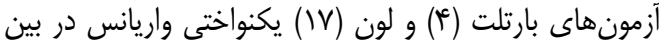

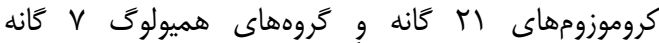

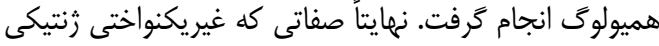

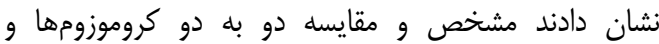

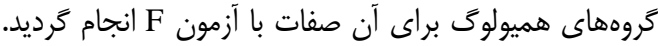
براى انجام تجزيههاى آمارى از نرمافزارهاى براى Minitab

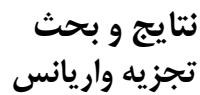

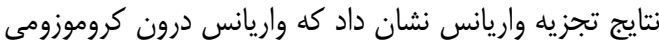

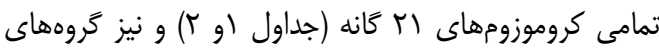

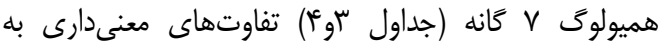

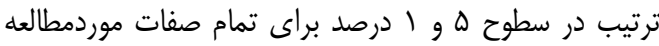

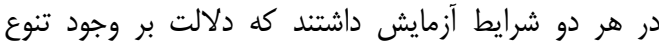

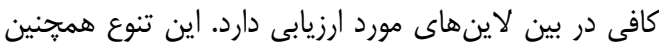

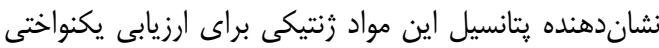

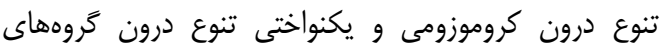

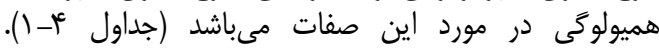

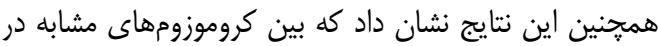

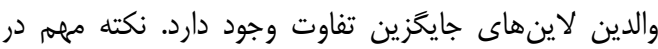

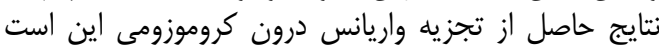

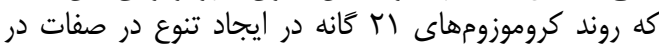

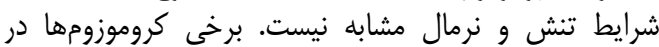

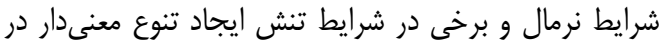

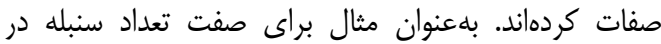

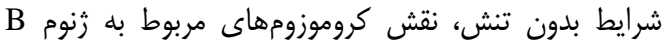

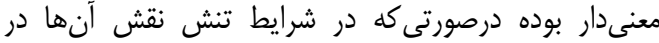

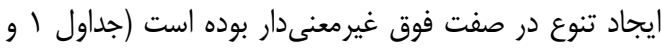


Tabel 1. Analyses of variance of intra chromosome for different traits in normal conditions

جدول ا- تجزيه واريانس درون كروموزومى براى صفات مختلف در شرايط نرمال A

\begin{tabular}{|c|c|c|c|c|c|c|}
\hline عملكرد دانه در بوته & شاخص برداشت & عملكرد بيولوزيك (كرم) & وزن هزار دانه (كرم) & تعداد دانه در سنبله & تعداد سنبله & كروههاى كروموزومى \\
\hline$\cdot 1 \cdot 9^{\mathrm{nS}}$ & $v / / v^{n s}$ & $r / V e^{n s}$ & $\cdot / \cdot v^{\mathrm{nS}}$ & $\mid A T / \Delta S^{\circ}$ & $r / \Delta \Lambda^{\mathrm{nS}}$ & IA \\
\hline.$/ \mathrm{r}^{\mathrm{ns}}$ & $1+9 / \Psi^{* *}$ & $91 \cdot f^{*}$ & $\mid r / r g^{\mathrm{ns}}$ & $\Lambda V / r \Delta^{\mathrm{ns}}$ & $\varphi / . .^{*}$ & rA \\
\hline$\cdot / N^{* *}$ & $\mid r v / q r^{*}$ & $r / .{ }^{\mathrm{ns}}$ & $1 / / \Delta H^{n s}$ & $r . . / W^{\mathrm{ns}}$ & $r / \cdot \Lambda^{\mathrm{ns}}$ & rA \\
\hline$\cdot / \Lambda^{\mathrm{ns}}$ & $F \mid r / \Delta \Lambda^{* *}$ & $r / \leftarrow \Delta^{\mathrm{ns}}$ & $\Delta / q^{n s}$ & $r V / r r^{n s}$ & $\Delta / r \Delta^{*}$ & FA \\
\hline$\cdot / \pi e^{\mathrm{ns}}$ & $r \Delta \omega / q)^{\mathrm{ns}}$ & $\cdot / \Lambda r^{\mathrm{ns}}$ & $1 . / 18^{\mathrm{ns}}$ & $r \cdot r / Q q^{*}$ & $r / \cdot \lambda^{\mathrm{ns}}$ & $\Delta \mathrm{A}$ \\
\hline$\cdot / \cdot r^{\mathrm{ns}}$ & $18 \% / 90^{* *}$ & $r / r r^{n s}$ & $\mid g / F V^{* *}$ & $\Lambda) / \Delta r^{n s}$ & $r / r r^{n s}$ & $9 \mathrm{~A}$ \\
\hline $1 / \kappa^{* * *}$ & $r / \propto v^{\mathrm{ns}}$ & $q / r \vee^{* *}$ & $r \omega / 1^{* *}$ & $\Lambda / \wedge^{\mathrm{ns}}$ & $N \cdot \Lambda^{*}$ & VA \\
\hline $1 / \Delta r^{n s}$ & $1 f 4 / v^{n s}$ & $r F / \& \omega^{* *}$ & $\kappa / F \Lambda^{\mathrm{ns}}$ & $r \Delta / / \varphi^{\mathrm{ns}}$ & $r \cdot 10 \Lambda^{* *}$ & IB \\
\hline.$|\Delta|^{* *}$ & $\mid \pi N / \Delta \Delta^{\mathrm{ns}}$ & $r / \mu r^{\mathrm{ns}}$ & $M N / g r^{* *}$ & $r \cdot N / \Delta \Delta^{* *}$ & $1 / \wedge^{\mathrm{ns}}$ & rB \\
\hline$r / .^{* *}$ & $r \Lambda I / V^{* * *}$ & $1 / 48^{* *}$ & $\cdot \mid q r^{\mathrm{ns}}$ & $\mid \varepsilon \mathrm{V} / \cdot \mathrm{r}^{* * *}$ & $r / v Q^{* *}$ & ${ }^{\mu} \mathrm{B}$ \\
\hline $1 / v^{\mathrm{ns}}$ & $\mid \mathrm{V} / \mathrm{Aq}^{* *}$ & $9 / \Upsilon^{\text {ns }}$ & $\Delta F / T I^{* *}$ & $11 N / F v^{*}$ & $\mathrm{~V} / \Delta \Lambda^{* *}$ & $\mathrm{FB}$ \\
\hline$\cdot / \mathrm{Fv}^{\mathrm{ns}}$ & $1.9 / V r^{\mathrm{ns}}$ & $1 \% / r \varepsilon^{* * *}$ & $8 / r q^{*}$ & $\operatorname{lvg} / \wedge^{* *}$ & $1 \cdot 10 \Lambda^{* *}$ & $\Delta \mathrm{B}$ \\
\hline $1 / 1^{\mathrm{ns}}$ & $r \mid q / q^{n s}$ & $\Delta / q^{\mathrm{ns}}$ & $11 / 99^{*}$ & $\Delta q / \cdot \varphi^{*}$ & $1 . / v \Delta^{* * *}$ & $9 \mathrm{~B}$ \\
\hline$r / .9^{* *}$ & $1.9 / 1 r^{*}$ & $M / Y q^{* *}$ & $\varphi / \mathcal{N} \varepsilon^{\mathrm{ns}}$ & TrT/Kr* & $9 / \vee \Delta^{*}$ & VB \\
\hline$T / 1 Q^{\mathrm{ns}}$ & $\Lambda \cdot / r^{\mathrm{ns}}$ & $q / \Delta f^{\mathrm{ns}}$ & $r \cdot / \mu e^{* *}$ & $r \cdot 1 \cdot r^{\text {ns }}$ & $\Delta / \Delta \Lambda^{\mathrm{ns}}$ & ID \\
\hline.$/ \mathrm{r}^{\mathrm{ns}}$ & $\varphi \cdot 9 / 4 V^{* *}$ & $V / \Delta r^{n s}$ & $9 \vee / \wedge 9^{* *}$ & $\mid G \Gamma / A r^{*}$ & $F / V \Delta^{\mathrm{ns}}$ & rD \\
\hline $1 / \Delta \Delta^{* *}$ & $\mid \Delta V / F r^{n s}$ & $r / 9 \Delta^{\mathrm{ns}}$ & $r / v q^{n s}$ & $\mid r F / \Delta V^{*}$ & $\Delta / \cdot \Lambda^{\mathrm{ns}}$ & rD \\
\hline$r / r^{* * *}$ & $M \cdot / r v^{*}$ & $\mid f / g e^{* *}$ & $9 / 9 V^{*}$ & $r / \Delta r^{n s}$ & $N / 1^{*}$ & ED \\
\hline$\cdot / / r^{\mathrm{ns}}$ & $|F / \lambda|^{\mathrm{ns}}$ & nسrss & $\Delta / 1 Q^{\mathrm{ns}}$ & ST/TF* & $\mathrm{V} / .^{\mathrm{ns}}$ & $\Delta \mathrm{D}$ \\
\hline$\cdot / 1 q^{\mathrm{ns}}$ & $r N / T^{n s}$ & $F / r^{*}$ & $11 / r)^{*}$ & $q F / \Delta q^{*}$ & $r / .{ }^{\mathrm{ns}}$ & $9 \mathrm{D}$ \\
\hline $1 / \mu F^{* *}$ & $\left\lceil\Lambda \kappa / \leftarrow \Lambda^{* * *}\right.$ & $1 / r^{*}$ & $r \cdot / \Lambda^{* * *}$ & $\Delta H^{\prime} / Y^{* * *}$ & $\Delta / \mathrm{HT}^{* * *}$ & VD \\
\hline
\end{tabular}

جدول r- تجزيه واريانس درون كروموزومى براى صفات مختلف در شرايط تنش خشكى A Tabel 2. Analyses of variance of intra chromosome for different traits in water stress conditions

\begin{tabular}{|c|c|c|c|c|c|c|}
\hline 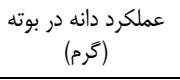 & شاخص برداشت & $\begin{array}{c}\text { عملكرد بيولوزيك } \\
\text { (كريك }\end{array}$ & وزن هزار دانه (كَرم) & تعداد دانه در سنبله & تعداد سنبله & كروههاى كروموزومى \\
\hline & & & & & & IA \\
\hline$\cdot / \mathrm{r}^{\mathrm{ns}}$ & $N \varepsilon / \pi r^{*}$ & $r / T \kappa^{n s}$ & $\Gamma / \widetilde{\sim} \omega^{n s}$ & $V M / Y e^{m^{*}}$ & $1 / \cdot n^{\mathrm{ns}}$ & rA \\
\hline$\cdot / 1 \mathrm{f}^{\mathrm{ns}}$ & $|V \omega /|^{* * *}$ & $11 / r^{*}$ & $1 \cdot 109^{\mathrm{ns}}$ & $r F / K \varepsilon^{n s}$ & $1 / 4 e^{\mathrm{ns}}$ & rA \\
\hline$\cdot|r|^{* *}$ & $190 / .1^{*}$ & $r / r r^{*}$ & $r r / \wedge q^{n s}$ & $\Delta / \bar{q} V^{\mathrm{ns}}$ & $1 / \Delta \Lambda^{\mathrm{ns}}$ & FA \\
\hline$\cdot / / v^{\mathrm{ns}}$ & $\mid V r / A e^{*}$ & $9 / 1 r^{*}$ & $T Y / D^{* *}$ & $r V / M^{*}$ & $\mu / \wedge^{* *}$ & $\Delta \mathrm{A}$ \\
\hline$\cdot / r 8^{\mathrm{ns}}$ & 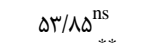 & $\Delta / \Delta 1^{\mathrm{ns}}$ & $\mid Q / f v^{\mathrm{ns}}$ & 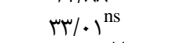 & $r / r^{* * *}$ & $\begin{array}{ll}\Delta \mathrm{A} \\
\varepsilon \mathrm{A}\end{array}$ \\
\hline$\cdot 1 \cdot 8^{\mathrm{ns}}$ & $19 \% / r Q^{* * *}$ & $1 / q^{\mathrm{ns}}$ & $f / \mu)^{n s}$ & $\mid r N / M^{* * *}$ & $s / r \Delta^{*}$ & $\begin{array}{l}7 \mathrm{~A} \\
\mathrm{y}\end{array}$ \\
\hline$\cdot|\Delta|^{* * *}$ & $\Delta N / r^{* * *}$ & $r / \Lambda r^{* *}$ & $\Delta / \kappa^{*} *$ & $V w / \wedge v^{n s}$ & $r / \Delta \Lambda^{\mathrm{ns}}$ & VA \\
\hline$\cdot 1 \cdot \mathrm{r}^{\mathrm{ns}}$ & $\Lambda \cdot / 1 *^{\mathrm{ns}}$ & $r / .^{\mathrm{ns}}$ & $1 / \cdot 1^{\mathrm{ns}}$ & $\cdot \mid \& 8^{\mathrm{ns}}$ & $1 / \wedge^{\mathrm{ns}}$ & IB \\
\hline$r / r^{* *}$ & $19 v \cdot / / f^{* *}$ & $r / F V^{*}$ & $\Delta T / T F^{* *}$ & $99 / r^{*}$ & $\Delta / r \omega^{*}$ & rB \\
\hline $1 / \Delta V^{* *}$ & YAY/90 ${ }^{* *}$ & $T V / \Psi \Lambda^{* *}$ & $\mid r / 9 q^{* *}$ & 1./r & $\cdot / \Delta \Lambda^{\mathrm{ns}}$ & ${ }^{\mathrm{B}}$ \\
\hline$\cdot / r^{n s}$ & $99 / 4^{*}$ & $9 / 99^{*}$ & $11 / \mathrm{qq}^{*}$ & $|r / \cdot|^{\mathrm{ns}}$ & $\cdot / V \Delta^{\mathrm{ns}}$ & $\mathrm{FB}_{\mathrm{B}}$ \\
\hline$\cdot / \Delta \mathrm{r}^{\mathrm{ns}}$ & $\Delta N / v \varepsilon^{n s}$ & $\mathrm{~V} / \wedge^{* *}$ & $\mid \varepsilon / \Psi^{* * *}$ & $|r| /\left.q\right|^{\mathrm{ns}}$ & $\cdot / r \Delta^{\mathrm{ns}}$ & $\Delta \mathrm{B}$ \\
\hline $1 / 1^{\mathrm{ns}}$ & $\mid \pi F / \Psi^{* * *}$ & $r / q \varepsilon^{\mathrm{ns}}$ & $\left.r \Delta / \phi^{*}\right)^{* *}$ & $r / \mathcal{N}^{\mathrm{ns}}$ & $r / r \Delta^{*}$ & $\Leftrightarrow \mathrm{B}$ \\
\hline $1 / / r^{* *}$ & $r \% / .9^{\text {ns }}$ & $1 . / 99^{* *}$ & $9 / 9 \Delta^{\mathrm{ns}}$ & $r q / v^{n s}$ & $91 \cdot 1^{\mathrm{ns}}$ & VB \\
\hline$\cdot 1 \cdot 8^{\mathrm{ns}}$ & $\Lambda \varepsilon / q q^{\mathrm{ns}}$ & $\cdot / \cdot^{\mathrm{ns}}$ & $F \Delta / \uparrow \varepsilon^{* *}$ & $r M / / 8^{* *}$ & $91 \cdot 1^{\mathrm{ns}}$ & VB \\
\hline $.1 \cdot 8^{\mathrm{ns}}$ & rag/As** & $r / r \Lambda^{\mathrm{ns}}$ & $r \Delta / T^{* *}$ & $194 / . r^{* * *}$ & . & ID \\
\hline.$/ 11^{*}$ & $r N / 9 e^{\mathrm{ns}}$ &.$|9|^{\mathrm{ns}}$ & $9 / \varphi^{n s}$ & 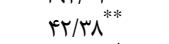 & $1 / V \Delta^{\mathrm{ns}}$ & TD \\
\hline$\cdot / r^{*}$ & $\Gamma \xi Q / \cdot \Gamma^{* * *}$ & $\cdot / .^{\text {ns }}$ & $r \cdot / . r^{* * *}$ & $|1 V / 9|^{*}$ & $r / \cdot \Lambda^{\mathrm{ns}}$ & rD \\
\hline$\cdot 1 \cdot \mathrm{n}^{\mathrm{ns}}$ & $99 / v^{n s}$ & $\Delta / \mathrm{V}^{\mathrm{ns}}$ & $\cdot|<|^{\mathrm{ns}}$ & $r 9 \mathrm{~V} / \mathrm{gr}^{* * *}$ & $1 / \cdot^{\mathrm{ns}}$ & ${ }^{E D}$ \\
\hline $.1 .9^{\mathrm{ns}}$ & $S N / T^{\mathrm{ns}}$ & $\Delta / q^{\mathrm{ns}}$ & $\mathrm{rV} / \cdot \mathrm{V}^{* *}$ & $\mathbb{N} / \mathrm{A}^{\mathrm{ns}}$ & $r / r r^{n s}$ & $\Delta \mathrm{D}$ \\
\hline \multirow[t]{2}{*}{.$/ W{ }^{* * *}$} & rצY/AV** & $1 / \Delta \varepsilon^{* *}$ & $r / \Psi^{n s}$ & $\mid r / s \varepsilon^{\mathrm{ns}}$ & $\varphi / . . *$ & $9 \mathrm{D}$ \\
\hline & & & & & & VD \\
\hline
\end{tabular}




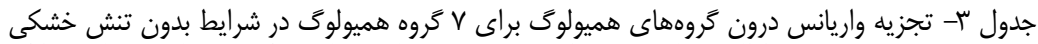
Tabel 3. Analyses of variance of intra hemeologous for 7 homologous groups in normal conditions

\begin{tabular}{|c|c|c|c|c|c|c|}
\hline 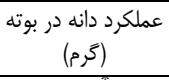 & شاخص برداشت & $\begin{array}{c}\text { عملكرد بيولوزيك } \\
\text { (كرم) }\end{array}$ & وزن هزار دانه (كرم) & تعداد دانه در سنبله & تعداد سنبله & كروههاى كروموزومى \\
\hline $1 / \Delta f^{\prime \prime}$ & $r \cdot \Delta / r \mu$ & $11 / 9 f^{* \cdots}$ & $\mid f / K f^{* \pi}$ & $99 / 49^{\cdots+7}$ & $q / \mu \varphi^{* w^{*}}$ & 1A1B1D \\
\hline.$/ \mu \digamma^{* * *}$ & 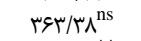 & $r / / k^{n s}$ & SV/r.*** & $\mid f \leftarrow / v \varepsilon^{* *}$ & $1 / 98^{\mathrm{ns}}$ & $2 \mathrm{~A} 2 \mathrm{~B} 2 \mathrm{D}$ \\
\hline $1 / r v^{* *}$ & 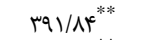 & $r / . \Delta^{*}$ & $N / \Delta V^{n s}$ & $\operatorname{QQT} / \mathrm{r}^{* *}$ & $r / l .{ }^{\mathrm{ns}}$ & 3A3B3D \\
\hline$r / 9^{* *}$ & TYN/AQ* & $N / r^{* * *}$ & $r \cdot / r \Lambda^{* *}$ & 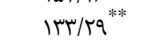 & $N / \Delta f^{* *}$ & $4 \mathrm{~A} 4 \mathrm{~B} 4 \mathrm{D}$ \\
\hline$\cdot / \Delta V^{*}$ & $9 \cdot 190^{\mathrm{ns}}$ & $F / M^{* *}$ & $g / \Delta S^{*}$ & $r \Delta / \Delta q^{n s}$ & $r / \Delta \cdot{ }^{n s}$ & $5 \mathrm{~A} 5 \mathrm{~B} 5 \mathrm{D}$ \\
\hline.$/ 99^{* *}$ & $1 Q \Delta / 9 \varphi^{* *}$ & $1 / r^{n s}$ & $\mid F / \cdot r^{* * *}$ & $\mid T V / \Delta V^{* *}$ & $r / \Lambda r^{\mathrm{ns}}$ & $6 \mathrm{~A} 6 \mathrm{~B} 6 \mathrm{D}$ \\
\hline$r / \cdot r^{* *}$ & $\operatorname{rIV} / 90^{* *}$ & $1 \Gamma / \Delta q^{* *}$ & $r \Delta / \Lambda r^{* *}$ & $r \mu / \Delta T^{* *}$ & $r / \mathscr{A} V^{*}$ & 7A7B7D \\
\hline
\end{tabular}

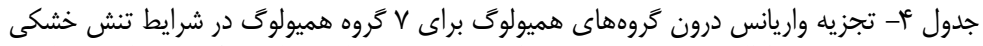
Tabel 4. Analyses of variance of intra hemeologous for 7 homologous groups in water stress conditions

\begin{tabular}{|c|c|c|c|c|c|c|}
\hline عملكرد دانه در بوته (كرم) & شاخص برداشت & 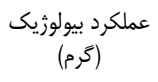 & وزن هزار دانه (كَرم) & تعداد دانه در سنبله & تعداد سنبله & كروههاى كروموزومى \\
\hline.$/ 1 r^{\mathrm{nS}}$ & $\mathcal{M} / \mathcal{F Y}^{\mathrm{nIS}}$ & $1 / 91^{1 \mathrm{nS}}$ & $T \cdot / T^{*}$ & $\mu F / \wedge r^{\mathrm{nS}}$ & $r / \backslash V^{\mathrm{ns}}$ & 1A1B1D \\
\hline $1 / 9 r^{* *}$ & $|Y 8| / 99^{* *}$ & $4 / 99^{* *}$ & $f \Delta / q . * *$ & $119 / v^{* *}$ & $r / q^{* * *}$ & $2 \mathrm{~A} 2 \mathrm{~B} 2 \mathrm{D}$ \\
\hline.$/ 9 \Lambda^{* *}$ & $\mathrm{~F} \cdot / \mathrm{Vq} \mathrm{q}^{* *}$ & $1 . / 9 e^{* *}$ & $\mid \varepsilon / r \Delta^{* *}$ & $9.119^{* *}$ & $1 /{ }^{n}{ }^{\mathrm{ns}}$ & $3 \mathrm{~A} 3 \mathrm{~B} 3 \mathrm{D}$ \\
\hline$\cdot 1 \Delta \Delta^{* *}$ & $91 / 9^{* *}$ & $N / r^{*}$ & $r \varepsilon / \cdot V^{* * *}$ & $1 F / 90^{*}$ & $1 / 91^{\mathrm{ns}}$ & $4 \mathrm{~A} 4 \mathrm{~B} 4 \mathrm{D}$ \\
\hline$\cdot / r^{\text {ns }}$ & $r T / \mu^{n s}$ & $1 / 19^{\mathrm{ns}}$ & $\mid r / v \varepsilon^{* *}$ & $4 F / 9 v^{n s}$ & $r / \Delta V^{*}$ & 4A4B4D \\
\hline.$/ \Lambda^{* *}$ & $1 \pi \cdot / M^{* *}$ & $r / r r^{\text {ns }}$ & $r \cdot / V \Lambda^{* *}$ & १1/9.** & $r / T{ }^{* *}$ & 5A5B5D \\
\hline.$/ \wedge V^{* *}$ & $\mid Q T / 9 \omega^{* *}$ & $\kappa / \mu^{* *}$ & $9 / \Delta V^{* *}$ & $F N / V \Delta^{\mathrm{ns}}$ & $r / \cdot r^{\mathrm{ns}}$ & $6 \mathrm{~A} 6 \mathrm{~B} 6 \mathrm{D}$ \\
\hline & & & & & & 7A7B7D \\
\hline
\end{tabular}

جدول ه- نتايج آزمون يكنواختى واريانس بين گروههاى كروموزومى براى صفات مورد بررسى" Tabel 5. The results of homogeneity analysis of variance among chromosome groups for the studied traits

\begin{tabular}{|c|c|c|c|c|c|c|c|c|}
\hline \multicolumn{6}{|c|}{ صفات } & & \multirow[b]{2}{*}{ آزمون } & \multirow[b]{2}{*}{ شرايط } \\
\hline 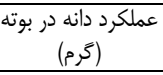 & شاخص & بيولوزيك (كمرم) & وزن هزار (كرم) & تعداد دانه در & تعداد سنبله & & & \\
\hline$\Lambda r / \Delta V$ & Tr/AT & 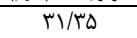 & Fハ/ノ9 & $T V / r F$ & $r+/ I I$ & آماره آزمون & \multirow{2}{*}{ بارتلت } & \multirow{4}{*}{ تنش بدون } \\
\hline.$/$. & r & $\cdot 1 \cdot \Delta$ &.$/$. & 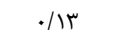 & $\cdot / F \Delta$ & سطح معنى دارى & & \\
\hline $1 / 9 V$ & - & $1 / .9$ & r/ar & $r / 1 \varepsilon$ & /9V & آماره آَزمون & \multirow[b]{2}{*}{ ل لون } & \\
\hline $.1 \cdot f$ &.$/ 17$ & عس/. & 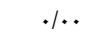 & $\cdot 1 \cdot+$ &.$/ 19 q$ & سطح معنى دارى & & \\
\hline $1 \cdot r / f \Delta$ & $\mathrm{VW} / \mathrm{Vg}$ & $V E / T r$ & $f 1 / r q$ & $r V / g r$ & $19 / 94$ & أماره آَزمون & \multirow{2}{*}{ بارتلت } & \multirow{4}{*}{ تنش } \\
\hline$\cdot / \cdot$ &.$/$. &.$/$. & r./.r &.$/ \cdot 1$ & .199 & سطح معنىدارى & & \\
\hline$r / \Lambda$. & $r / .9$ & $1 / \Delta r$ & $T / M r$ & $1 / 9 r$ & $1 / \cdot r$ & آماره آزمون & \multirow[b]{2}{*}{ لون } & \\
\hline .1. &.$/$. & $\cdot 1 \cdot 1$ & $.1+.1$ & $\cdot 1 \cdot \Delta$ & . Mf & سطح معنى دارى & & \\
\hline
\end{tabular}

جدول צ- نتايج آزمون يكنواختى واريانس بين گروههاى هميولوگ براى صفات مورد بررسى Tabel 6. The results of homogeneity analysis of variance among hemeologous groups for the studied traits

\begin{tabular}{|c|c|c|c|c|c|c|c|c|}
\hline \multicolumn{6}{|c|}{ صفات } & & \multirow[b]{2}{*}{ آزمون } & \multirow[b]{2}{*}{ شآزمايش } \\
\hline عملكرد دانه در بوته & برداشت & 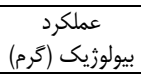 & دانه (كرم) & تعداد دانه در & تعداد سنبله & & & \\
\hline$T r / V T$ & $V / r q$ & $\mid F / \& D$ & $T / / V$ & $1 \cdot / \mathrm{VA}$ & $V / V V^{f}$ & آماره آزمون & \multirow[t]{2}{*}{ بارتلت } & \multirow{4}{*}{ 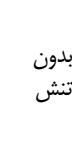 } \\
\hline $.1 . .1$ &.$/ 4 q$ & .1 .4 & $.1 . .1$ & .1 .9 & . & سطح معنىدارى & & \\
\hline $1 / V$ & $1 / \cdot 1$ & $1 / \Delta 1$ & r/q4 & $1 / \Delta \Lambda$ & $r / \uparrow \Delta$ & آماره آزمون & \multirow[b]{2}{*}{ لون الون } & \\
\hline . &.$/ r v$ &.$/ 11$ &.$/$. &.$/ 10$ & r./. & سطح معنى دارى & & \\
\hline$r q / M$ & $9 \cdot 190$ & $I T / Q T$ & $9 / 4 \wedge$ & $F / 4$ & . & آماره آزمون & \multirow{2}{*}{ بارتلت } & \multirow{4}{*}{ تنش } \\
\hline.$/$. &.$/$. & $.1 \cdot 0$ &.$/ 10$ & Tat &.$/ 99$ & سطح معنى داري & & \\
\hline$\Gamma / \Delta \omega$ & 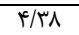 & $1 / . \mu$ & $1 / \uparrow \Delta$ &.$/ \mathrm{Vq}$ &.$/ T \Lambda$ & آماره آزمون & \multirow{2}{*}{ لون } & \\
\hline .1 .4 &.$/$. &.$/ 41$ &.$/ r$ &.$/ \Delta \Lambda$ &.$/ 90$ & سطح معنى دارى & & \\
\hline
\end{tabular}


Tauschii و كو Talopes

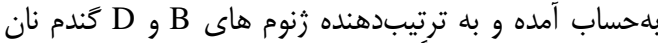

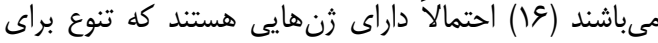

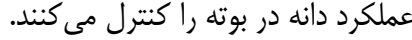

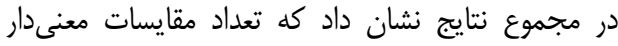

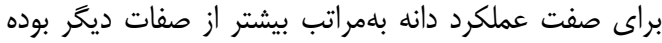

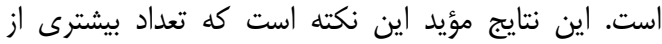

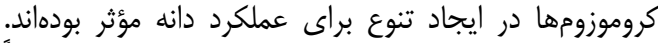

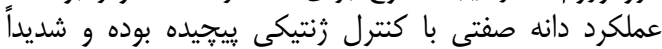

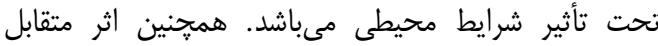

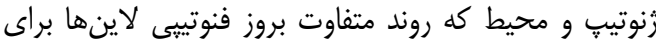

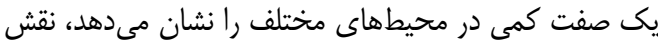

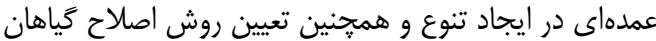

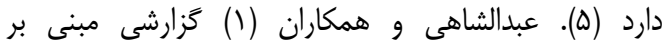

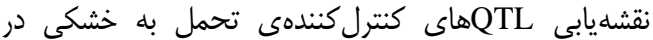

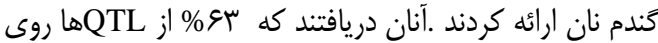

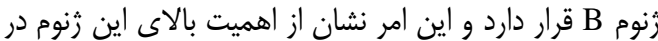

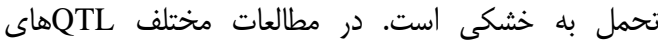

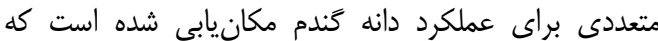

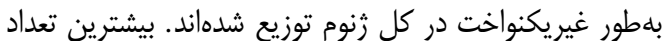

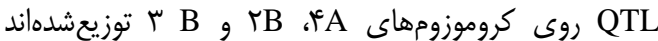

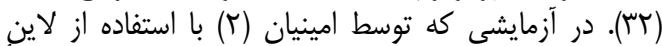

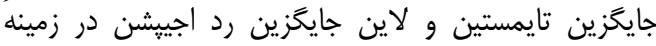

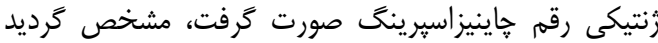

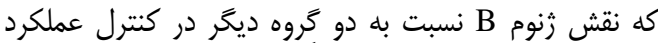

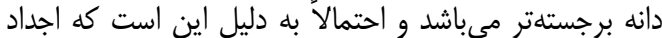

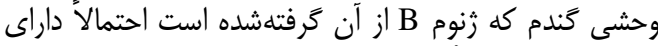

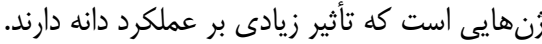
مقايسه دو بدوى واريانس تَروهائ كائ كروموزومى براني صفت وزن هزار دانه دوانه براى وزن هزار دانه مقايسه دو بدون دوانه واريانس درون درون

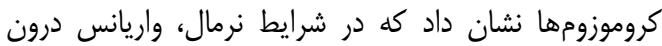

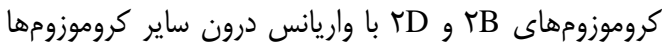

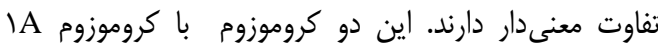

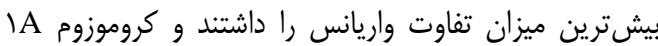

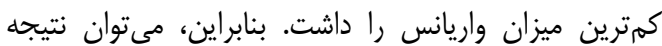

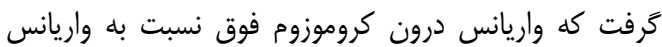

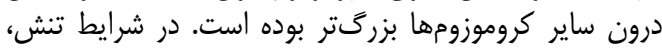

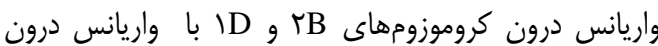

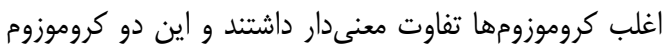

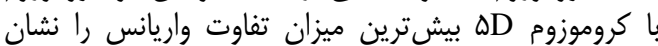

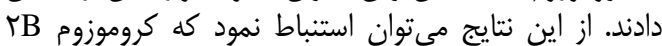

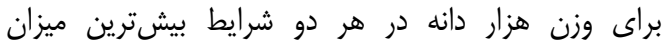

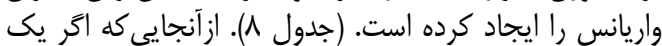

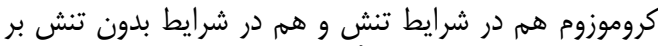

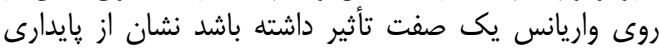

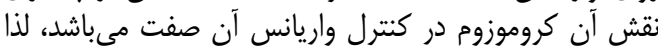
نقش كروموزوم TB در مقايسه با ساير كروموزومها بإيدارتر
با توجه به جدول (ه) و در مقايسه شرايط بدون تنش علش برد

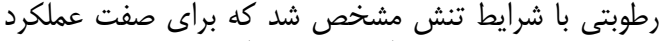

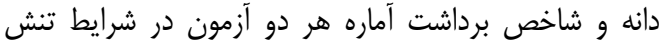

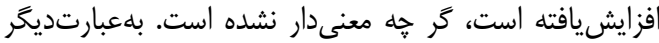

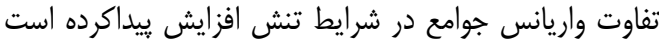

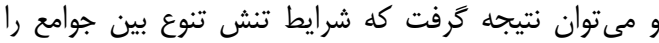

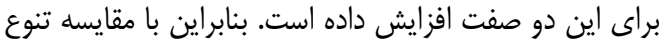

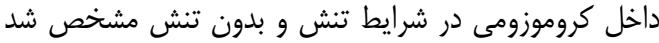

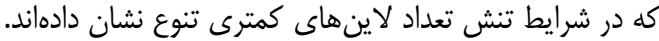

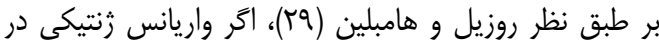

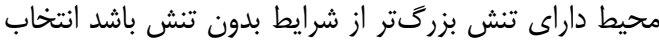

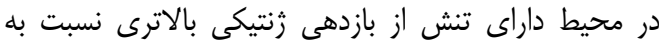

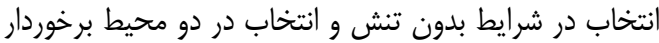

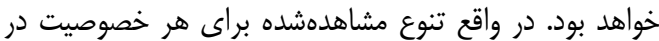

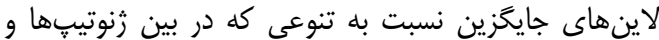

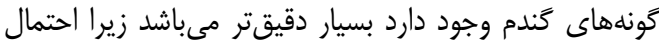

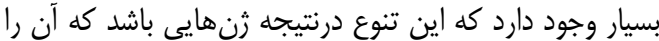

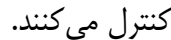

مقايسه واريانس دو بدنئ بدوى تروههاى كروموزومى براى صفت عملكرد دانه

ازآنجايى كه هم در شرايط تنش و و هم در شرايط بدون

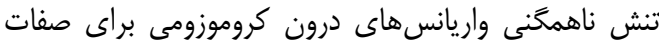

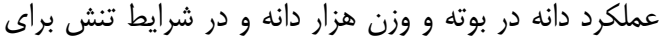

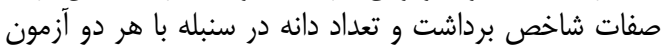

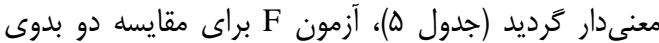

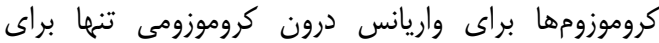
صفات فوق انجام شد.

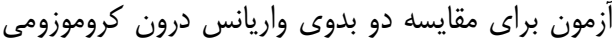

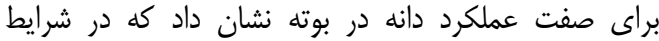

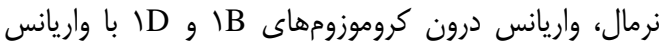

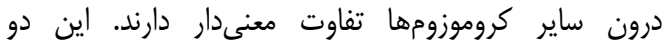

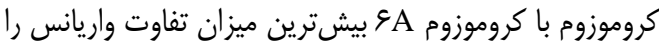

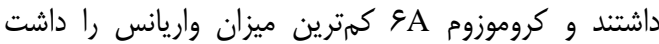

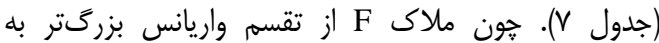

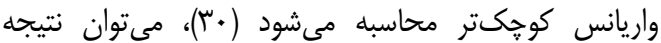

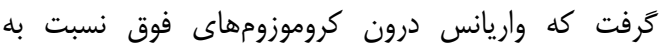

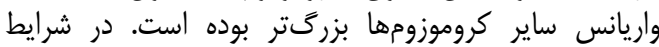

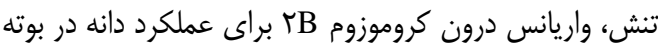

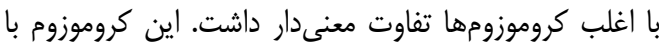

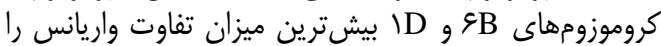

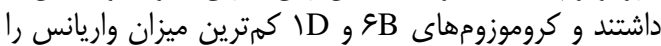

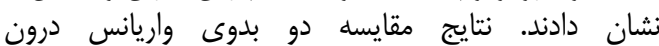

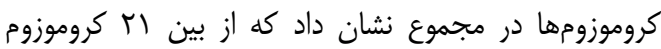

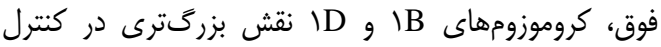
واريانس صفت عملكرد دانه در بوته دارند. بنابراين بيشترئرين

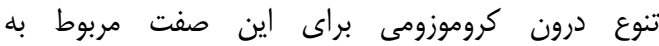

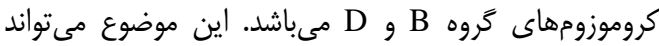
متخصصين اصلاح نباتات را به اين نكته متوجه سازد كه كَونه 
مF از تقسيم واريانس بزرگتر به واريانس كوخكتر محاسبه

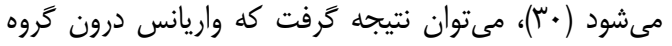

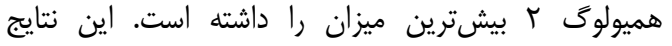

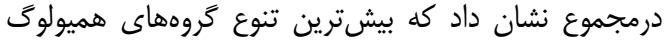

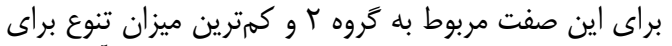

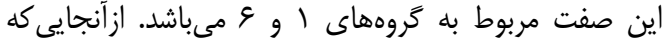

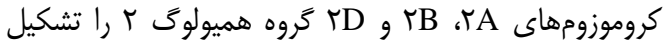

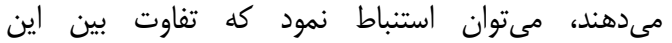

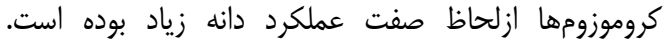

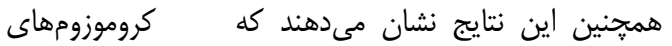

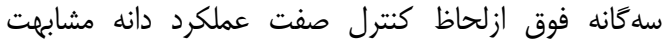

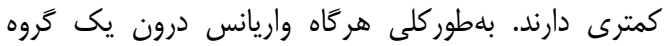

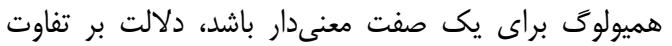

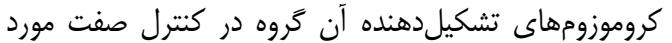

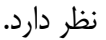

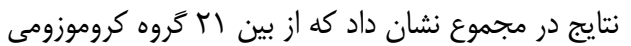

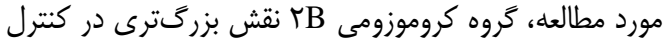

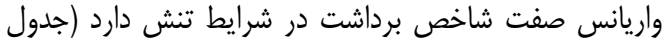

مقايسه دو بدوى واريانس درون كروموزومى براى صفت تعداد دانه در سنبله

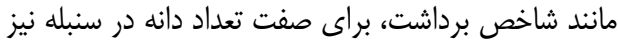

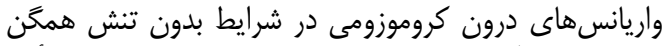

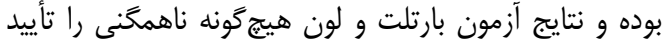

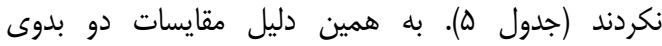

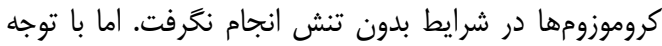

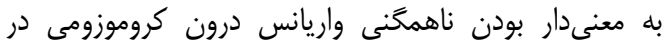

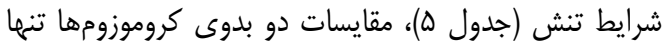

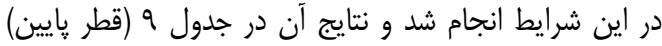

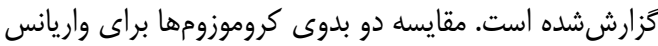

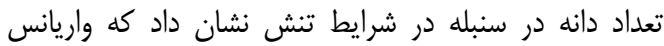

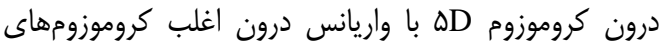

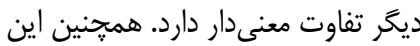

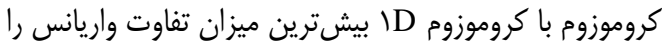

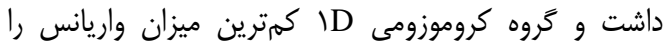

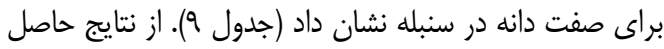

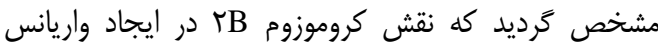

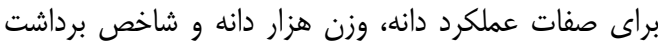

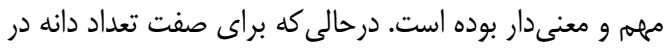

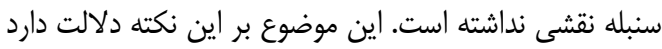

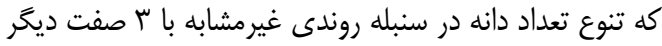

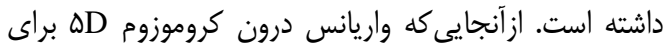

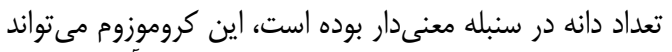

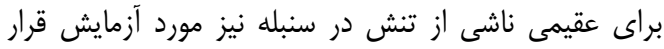

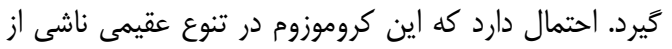
تنش در كَدم نقش داشته باشد (؟هار).

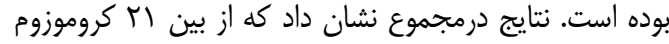

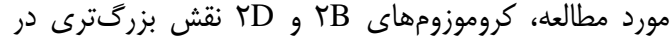

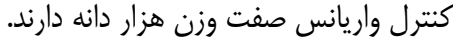
مقايسه دو بدوى واريانس درون درون كروموزومى برائ صفت شاخص برداشت

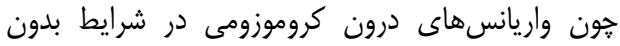

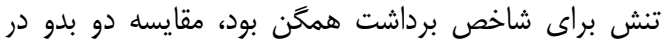

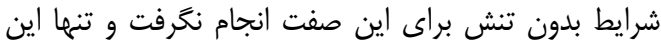

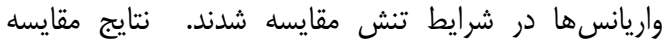

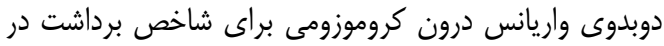

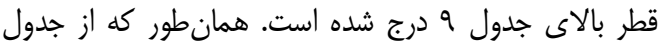

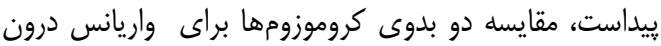

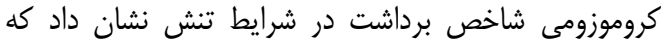

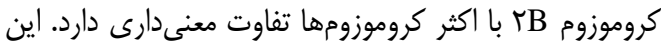

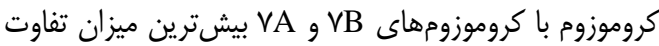

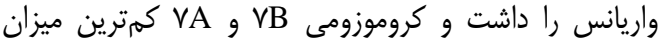
وراريانس درون كروموزومى را داشتند. بررسى يكنواختى واريانس دانى د درون َروههاى

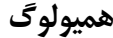

در اين تحقيق، هفت خروه هميولوَى در دو شرايط بدون

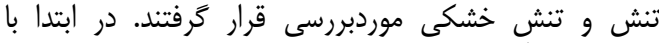

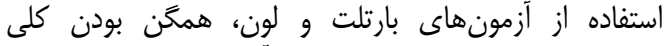

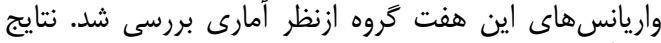

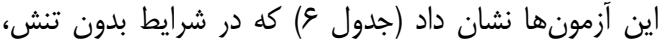

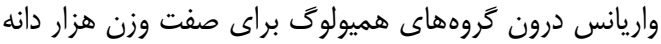

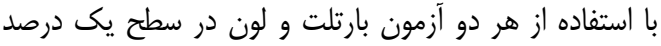

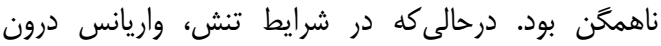

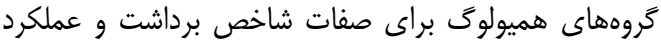

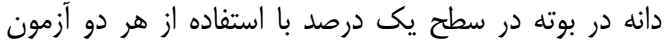

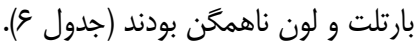

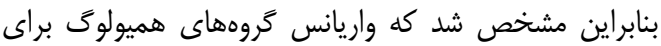

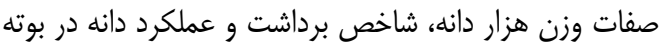

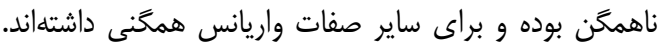

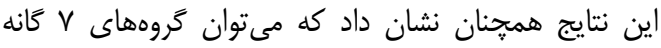

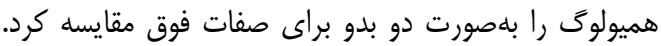

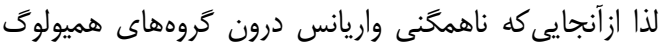

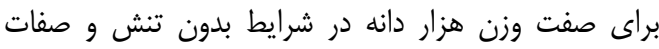

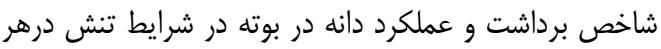

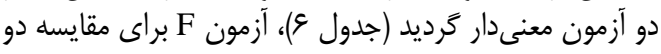

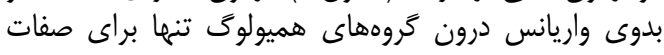
فوق انجام گرديد.

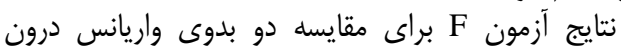

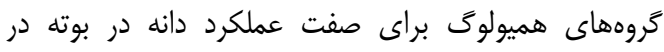

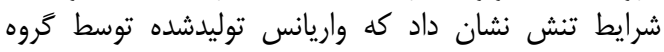

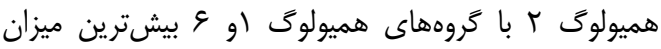
تفاوت واريانس را دارا بودند (جدول • (1). جون ملاك محاس محاسبه 
جدول V- مقايسه دو بدوى كروههاى كروموزومى براى واريانس درون كروموزومى صفت عملكرد دانه در شرايط بدون تنش (بالاى قطر) و در شرايط تنش (بايين قطر) Table 7. Comparison of two primitive chromosome groups for intra-chromosomal variance of grain yield with f test under stressless conditions (above diameter) and under

\begin{tabular}{|c|c|c|c|c|c|c|c|c|c|c|c|c|c|c|c|c|c|c|c|c|c|}
\hline VD & $9 \mathrm{D}$ & $\Delta \mathrm{D}$ & ${ }^{4} \mathrm{D}$ & rD & rD & ID & VB & $9 \mathrm{~B}$ & $\Delta \mathrm{B}$ & ${ }^{\circ} \mathrm{B}$ & ${ }^{\mathrm{B}} \mathrm{B}$ & TB & IB & $\mathrm{VA}$ & $9 \mathrm{~A}$ & $\Delta \mathrm{A}$ & ${ }^{r} \mathrm{~A}$ & rA & rA & IA & \\
\hline$r / V)^{\circ}$ & $9 / \mathrm{T}^{28}$ & $1 / \cdot v^{\mathrm{nis}}$ & $N / N^{* 3 *}$ & $\mathcal{L} / \sim^{\prime *}$ & $1 / \cdot r^{\text {ris }}$ & $1 . / .^{* * *}$ & $9 / 0^{\circ 8}$ & $8 / 10^{\circ \circ}$ & $T / \&\}^{n S}$ & $\Delta / T)^{*}$ & 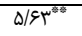 & $1 / \Delta)^{\mathrm{ns}}$ & $1 \cdot / 4 \Lambda^{\circ 9}$ & $\kappa / V^{*}$ & $T / V \cdot{ }^{n s}$ & $r / / r^{n s}$ & $1 / r^{\mathrm{nis}}$ & $r / q^{\mathrm{nIS}}$ & $1 / r \Delta^{\mathrm{ns}}$ & 1 & IA \\
\hline$\Delta / \cdot 1^{*}$ & $N / \Delta q^{* 83}$ & $1 / \% \Delta^{\mathrm{ns}}$ & $11 / \mathrm{N}^{* * a}$ & $\Delta / 9 .^{\circ b}$ & $1 / r^{n s}$ & $15 / \Delta^{80}$ & $N \mid 99^{\circ 3}$ & $9 / / v^{83}$ & $r / T^{*}$ & $\mathrm{~V} / \cdot \mathrm{r}^{\mathrm{mas}}$ & $\mathrm{V} / \mathrm{s} \cdot{ }^{* * *}$ & $r / \cdot M^{\mathrm{ns}}$ & $1 F / 1 f^{\circ *}$ & $s / \mu t^{* * *}$ & $\gamma / .^{n s}$ & $r / \Delta \gamma^{n s}$ & $1 / r^{n{ }^{n s}}$ & $r / \Delta \|^{\prime \prime}$ & 1 & $1 / 9 \mathrm{v}^{\mathrm{ns}}$ & rA \\
\hline $1 / F r^{n s}$ & $r / \& \Delta^{\text {ns }}$ & $r / . .^{\text {ns }}$ & $r / \% \Delta^{n s}$ & $1 / / \Lambda^{\text {ns }}$ & $r / 8 \Lambda^{\text {ns }}$ & $r / \Lambda \kappa^{*}$ & $r / \mathcal{F r}^{\mathrm{ns}}$ & $r / q)^{\text {ns }}$ & $1 / \cdot^{\mathrm{ns}}$ & $r / .^{\mathrm{ns}}$ & $r / / \gamma^{\mathrm{ns}}$ & $1 / 99^{n s}$ & $\kappa / \% r^{*}$ & $1 /(1)^{n s}$ & $\mathrm{~V} / \cdot \mathrm{r}^{\mathrm{r**}}$ & $1 / \pi r^{n s}$ & $r / 99^{\mathrm{ns}}$ & 1 & $1 / 9^{q^{n s}}$ & $1 / \Delta r^{\mathrm{ns}}$ & $r_{\mathrm{A}}$ \\
\hline$\Gamma / \Lambda *^{*}$ & $s \mid \Delta T^{\Delta s}$ & $1 / 1 .^{\mathrm{ns}}$ & $N / q r^{20 s}$ & $\Gamma / \leftarrow \Lambda^{n s}$ & $1 / \cdot^{\mathrm{ns}}$ & $1 . / \pi e^{s * n}$ & $\varepsilon / \Delta V^{\circ \circ}$ & $8 / 99^{\circ 0}$ & $r / \Delta \Lambda^{n s}$ & $\Delta / \Psi^{\prime \prime}$ & $\Delta / V^{\theta * s}$ & $1 / \Delta \Lambda^{\mathrm{ns}}$ & $1 . / V T^{s=8}$ & $\mid\left\langle/\left.\Lambda\right|^{\circ}\right.$ & $r / q r^{n s}$ & $r / \Lambda^{\mathrm{ns}}$ & 1 & $1 / v^{n s}$ & $1 / \wedge \gamma^{\mathrm{ns}}$ & $1 / / r^{\mathrm{ns}}$ & $r_{\mathrm{A}}$ \\
\hline $1 / N \Delta^{\mathrm{ns}}$ & $r / . .^{\text {ns }}$ & $1 / 9 \Lambda^{\mathrm{ns}}$ & $5 / 11^{*}$ & $r / \bullet^{\mathrm{ns}}$ & $r / q^{q^{n s}}$ & $F / V)^{*}$ & $r / \cdot r^{\mathrm{ss}}$ & $r / r \cdot{ }^{n s}$ & $1 / 18^{\mathrm{ns}}$ & $r / F \Delta^{\mathrm{ns}}$ & $r / q D^{\mathrm{ns}}$ & $1 / \mathrm{rn}^{\mathrm{ns}}$ & $r / q r^{*}$ & $r / r^{\mathrm{ns}}$ & $\Delta / V^{* * *}$ & 1 & $1 / \cdot 0^{\mathrm{ns}}$ & $1 / \wedge^{\mathrm{ns}}$ & $1 / 9 y^{n s}$ & $1 / 1 \Lambda^{\mathrm{ns}}$ & $\Delta \mathrm{A}$ \\
\hline $1.1 . .^{* *}$ & $\mid V / V^{* s}$ & $r / \Lambda q^{\text {ns }}$ & $\Gamma / \Delta \psi^{* \Delta}$ & $11 / v^{* * *}$ & $r / q Y^{n s}$ & $r \varepsilon / 9 Y^{\text {sit }}$ & $\mid V / /^{\mu^{* *}}$ & $\mid N / \Psi^{* \Delta *}$ & G/g & 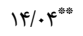 & $\mid \theta / / \Lambda^{* * \theta}$ & $\kappa / \backslash \varphi^{*}$ & $T N / T D^{s *}$ & $\mid r / s \mathrm{~V}^{\circ 8}$ & 1 & $r / r^{q^{n s}}$ & $r / T v^{\mathrm{ns}}$ & $1 / T \gamma^{\mathrm{ns}}$ & $1 / 18^{\mathrm{ns}}$ & $1 / 9 e^{\mathrm{ns}}$ & $9 \mathrm{~A}$ \\
\hline $1 / T v^{n s}$ & $1 / / 4^{\mathrm{ns}}$ & $\kappa / \mu^{\circ}$ & $1 / / s^{\mathrm{ns}}$ & $1 / \cdot v^{n s}$ & $r / \Lambda r^{*}$ & $r / 1 r^{\text {ns }}$ & $1 / r r^{\mathrm{ns}}$ & $1 / \kappa^{\mathrm{ns}}$ & $1 / 91^{\mathrm{ns}}$ & $1 / 1^{\mathrm{ns}}$ & $1 / r^{n s}$ & $r / \cdot \mathrm{r}^{\mathrm{ns}}$ & $r / \pi r^{\text {ns }}$ & 1 & $r / v)^{n s}$ & $1 / \Lambda^{\mathrm{ns}}$ & $1 / \pi \Delta^{\mathrm{ns}}$ & $r / / \mathrm{f}^{\mathrm{ns}}$ & $r / T r^{n s}$ & $1 / r^{n s}$ & $\mathrm{VA}$ \\
\hline$r / \Lambda r^{n s}$ & $1 / 9 \varphi^{\mathrm{ns}}$ & $9 / v V^{s s}$ & $1 / r^{\mathrm{ns}}$ & $r / 4 .{ }^{n s}$ & $1 . / \mathrm{V} \mathrm{N}^{* 0 *}$ & $1 / \cdot 0^{\mathrm{ns}}$ & $1 / / Y^{n s}$ & $1 /\left.\Delta\right|^{\mathrm{ns}}$ & $r / \pi \varphi^{\prime \prime}$ & $r / \cdot{ }^{\mathrm{ns}}$ & $1 / \mathcal{N}^{\mathrm{ns}}$ & S/NQ"* & 1 & $r / / v^{n s}$ & $1 / \pi \omega^{\mathrm{ns}}$ & $1 / / \mathrm{rn}^{\mathrm{ns}}$ & $1 / N \Delta^{\mathrm{ns}}$ & $1 / \cdot 1^{\mathrm{ns}}$ & $1 / v^{\mathrm{ns}}$ & $1 / \Delta \mathrm{s}^{\mathrm{ns}}$ & IB \\
\hline$r / 4 \cdot{ }^{n s}$ & $\varphi / \mu^{* s s}$ & $1 / k e^{\text {ns }}$ & $\Delta / 9 \Lambda^{\circ / \theta}$ & $r / A n^{n s}$ & $1 / \Delta Q^{n s}$ & $\mathbb{F} / 4 \Lambda^{\circ 8}$ & $r / \backslash q^{\prime \prime}$ & $r /\left.F\right|^{*}$ & $1 / \Delta q^{n s}$ & $r / T v^{\text {ns }}$ & $\Gamma / 9 \Delta^{*}$ & 1 & $1 Q / T T^{606}$ & $\mathrm{~V} / \cdot \mathrm{r}^{* * * *}$ & $19 / \cdot r^{\circ 8}$ & $N / T^{*}$ & $N / V \varphi^{\circ \theta}$ & $|Q| \cdot Y^{\Delta * \theta}$ & $18 / 19^{6 * t}$ & $q / \mathrm{v}^{908}$ & rB \\
\hline $1 / \Delta r^{n s}$ & $1 / 1 r^{\mathrm{ns}}$ & $\Delta / T \Delta^{\circ}$ & $V / \Delta \Delta^{\mathrm{nS}}$ & $1 / / q^{\mathrm{ns}}$ & $\Delta / v^{* \theta}$ & $1 / \mathrm{v}^{\mathrm{ns}}$ & $1 / / \mathrm{e}^{\mathrm{ns}}$ & $1 / T^{\mathrm{ns}}$ & $r / r^{q^{n s}}$ & $1 / \Lambda^{*}$ & 1 & $r / / \gamma^{\mathrm{ns}}$ & $\gamma / \cdot r^{\mu s}$ & $\pi / \pi \mathrm{n}^{\mathrm{ss}}$ & $N / \mathrm{N}^{\mathrm{s} * \mathrm{~s}}$ & $r / A f^{\circ}$ & $\kappa / \varphi^{*}$ & s/ar" & $V / \Delta \Delta^{\circ \Delta}$ & $r / \Delta T^{*}$ & rB \\
\hline$|/ F|^{n s}$ & $1 / r^{\mathrm{ns}}$ & $\Gamma / \mathcal{N}^{*}$ & $1 / / \wedge^{\mathrm{ns}}$ & $1 / 19^{\mathrm{ns}}$ & $\Delta / \Psi^{* \theta B}$ & 1/qr & $1 / r^{n^{n s}}$ & $1 / \Gamma^{n s}$ & $r / / r^{n s}$ & 1 & $r /\left.\Delta\right|^{*}$ & $V / 9 \cdot * *$ & $r / .{ }^{n s}$ & $1 / \cdot \Lambda^{\mathrm{ns}}$ & $r / \Delta \cdot{ }^{n s}$ & $1 / 9^{95}$ & $1 / 1 Q^{\mathrm{ns}}$ & $1 / 94^{\mathrm{ns}}$ & $r / 10^{\mathrm{ns}}$ & $1 / r^{q^{n s}}$ & ${ }^{4} \mathrm{~B}$ \\
\hline $1 /\left.\Delta\right|^{\mathrm{ns}}$ & $r / \Delta q^{n s}$ & $r / r q^{n s}$ & $\Gamma / \Delta \Delta^{\circ}$ & $1 / \mathrm{V}^{\mathrm{ns}}$ & $r / \Delta r^{\text {ns }}$ & $\psi / \cdot V^{*}$ & $r / \varphi^{\text {ns }}$ & $r / W^{\mathrm{ns}}$ & 1 & $r / r^{\mathrm{ns}}$ & $1 / r^{n s}$ & $\Gamma / V \Delta^{*}$ & $4 / .9^{* \prime}$ & $1 / \wedge \mathrm{n}^{\mathrm{ns}}$ & $\Delta / \cdot V^{*}$ & $r / \pi r^{\text {ns }}$ & $r / r r^{\mathrm{ns}}$ & $\% / . "$ & $F / \varphi^{*}$ & $r /\left.\right|^{\text {ns }}$ & $\Delta \mathrm{B}$ \\
\hline $1 / \wedge r^{n s}$ & $1 / \cdot r^{\mathrm{ns}}$ & $8 / N e^{\circ s}$ & $1 / r^{n s}$ & $1 / \Delta \Delta^{\mathrm{ns}}$ & $8 / 99^{* 8}$ & $1 / 4 v^{\mathrm{ns}}$ & $1 / \cdot \bullet^{\mathrm{ns}}$ & 1 & $1 . / 49^{* * t}$ & $\Delta / / \Upsilon^{*}$ & $\mid N / .^{* *}$ & $r q / T \varphi^{9 s}$ & $\Gamma / \Delta \Lambda^{\mathrm{nS}}$ & $\Delta / \Delta Q^{* * *}$ & $r / \bullet^{\mathrm{ns}}$ & $F / V r^{*}$ & $\kappa / 4 \mathcal{A}^{*}$ & $r / \varphi^{\mathrm{nS}}$ & $T / \mathrm{F}^{\mathrm{ns}}$ & $r / \cdot 1^{*}$ & $\varphi_{\mathrm{B}}$ \\
\hline $1 / \mathrm{Nr}^{\mathrm{ns}}$ & $1 / \cdot 1^{\mathrm{ns}}$ & $\Delta / 4 \Lambda^{5 s}$ & $1 / 4 \varphi^{*}$ & $1 / F \gamma^{\mathrm{ns}}$ & $8 / 90^{\circ 8}$ & $1 / \Delta n^{n s}$ & 1 & $11 / e^{a s}$ & $1 / 1 r^{\mathrm{ns}}$ & $T / T \mathrm{n}^{\mathrm{ss}}$ & $1 / \Delta)^{n s}$ & $r / r r^{n^{n s}}$ & $\kappa / \Delta S^{\prime \prime}$ & $r / 1 .{ }^{\text {ns }}$ & $\Delta / 99^{\circ / 4}$ & $r / \& q^{n s}$ & $r / \mathcal{Y} n^{\mathrm{ss}}$ & $r / \& q^{*}$ & $\mathrm{r} / 1 \mathrm{~A}^{*}$ & $r / q r^{n s}$ & VB \\
\hline$r / v \cdot{ }^{n s}$ & $1 / \Delta Y^{\mathrm{ns}}$ & $9 / \pi r^{\circ 00}$ & $1 / 10^{\mathrm{ns}}$ & $r / q^{q^{n s}}$ & $1 . / r^{* * *}$ & 1 & $1.19 \mathrm{~V}^{\circ \mathrm{sen}}$ & $1 / 1^{\mathrm{ns}}$ & $9 / \Delta)^{\cdots}$ & $r / 9 q^{*}$ & $18 / 19^{* 0 *}$ & $r \Delta / \& V^{\circ *}$ & $r / r r^{n s}$ & $\Delta / \cdot \Lambda^{\circ}$ & $1 / M^{n s}$ & $F / q^{\circ}$ & $r / v^{* \prime}$ & $r / \Lambda^{n s}$ & $r / \Lambda^{n s}$ & $r / 9 \varphi^{* \prime \prime}$ & ID \\
\hline$r / \Lambda r^{*}$ & $8 / \Delta \Delta^{* \Delta}$ & $1 / 1 \cdot \cdot^{\mathrm{ns}}$ & $N / 9 a^{* * *}$ & $\kappa / \Delta .^{*}$ & 1 & $1 / v r^{n s}$ & $8 / 19^{* 0 *}$ & $1 / 9)^{\mathrm{ns}}$ & $\Delta / \kappa^{* * \theta}$ & $r / V^{\mathrm{ns}}$ & $9 / \Delta 0^{* \infty}$ & $r \cdot / \Delta \mathrm{C}^{30 \mathrm{~B}}$ & $1 / \pi \Delta^{\mathrm{ns}}$ & $r / q r^{\text {ns }}$ & $1 / \cdot 1^{\mathrm{ns}}$ & $r / \mathcal{e r s}$ & $r / \mu^{\mathrm{ns}}$ & $1 / r r^{n s}$ & $1 / r \varphi^{n s}$ & $r / r^{\text {ns }}$ & TD \\
\hline $1 / / \Lambda^{\mathrm{ns}}$ & $1 / k \varepsilon^{\text {ns }}$ & $\% / \cdot \wedge^{\circ}$ & $r / .^{\mathrm{ns}}$ & 1 & $1 / \kappa \Psi^{n s}$ & $r / F \Lambda^{\mathrm{ns}}$ & $r / r \cdot *$ & $r / W^{r^{n s}}$ & $r / \Lambda r^{* s}$ & $1 / \wedge^{q^{n s}}$ & $\varepsilon / q^{* 08}$ & $14 / \pi 9^{96}$ & $1 / \cdot \bullet^{\mathrm{ns}}$ & $\gamma / \cdot \kappa^{\mathrm{ns}}$ & $1 / r^{n s}$ & $1 / r^{r^{n s}}$ & $1 / q^{e^{n s}}$ & $1 / \cdot 0^{\mathrm{ns}}$ & $1 / 1 \mathrm{n}^{\mathrm{ns}}$ & $1 / / v^{\mathrm{ns}}$ & ${ }^{r} \mathrm{D}$ \\
\hline$\Gamma / \Gamma^{n s}$ & $1 / r \gamma^{\mathrm{ns}}$ & $N / e^{* a *}$ & 1 & $1 / 1{ }^{\mathrm{ns}}$ & $1 / \Delta \Lambda^{n s}$ & $r / N^{n s}$ & $r / 9)^{*}$ & $r / .^{\text {ns }}$ & 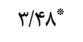 & $1 / N r^{n s}$ & $81 . r^{\circ 0^{\circ}}$ & $\mid r / \cdot V^{5 s}$ & $1 / 1 v^{n s}$ & $1 / \mathcal{N}^{\mathrm{ns}}$ & $1 / \epsilon^{\mathrm{ns}}$ & $1 / \Delta r^{\mathrm{ns}}$ & $1 / \mathrm{F}^{\mathrm{ns}}$ & $1 / 10^{\mathrm{ns}}$ & $1 / \pi \omega^{\mathrm{ns}}$ & $1 / r^{n s s}$ & ${ }^{\circ} \mathrm{D}$ \\
\hline$r / 48^{\circ}$ & $\Delta / q e^{\omega * s}$ & 1 & $1 / 1 r^{\mathrm{ns}}$ & $1 / \pi e^{n^{n s}}$ & $1 / \mathrm{N}^{\mathrm{ns}}$ & $r / \cdot q^{\mathrm{ns}}$ & $r / \mathscr{\varepsilon} q^{*}$ & $r / 4 \cdot{ }^{n s}$ & $r / \cdot \Lambda^{\text {ns }}$ & $1 / \Delta r^{n s}$ & $\Delta / \Psi^{* \Delta s}$ & $11 / \Delta 9^{\theta *}$ & $1 / r^{n s}$ & $1 / 9 \mathrm{e}^{\mathrm{ns}}$ & $1 / 9 \omega^{\mathrm{ns}}$ & $1 / \mathrm{rq}^{\mathrm{ns}}$ & $1 / r r^{n s}$ & $1 / r^{n s}$ & $1 / 4 r^{n s}$ & $1 / \Lambda^{\mathrm{ns}}$ & $\Delta \mathrm{D}$ \\
\hline $\mathrm{I} / \mathrm{r} \mathrm{r}^{\mathrm{ns}}$ & 1 & $1 / r^{n}{ }^{\mathrm{ns}}$ & $1 / 10^{\mathrm{ns}}$ & $1 / \cdot \mathrm{ers}^{\mathrm{ns}}$ & $1 / r v^{\mathrm{ns}}$ & $r / r \Lambda^{\mathrm{ns}}$ & $\kappa / \mathcal{E N}^{\circ}$ & $r / \mathcal{G} r^{\mathrm{ns}}$ & $\varphi / . . *$ & $1 / 9 \gamma^{\mathrm{ns}}$ & $8 / 9 T^{5 * a}$ & $15 / 99^{50}$ & $1 / \cdot r^{n s}$ & $r / r^{n s}$ & $1 / T \mathrm{r}^{\mathrm{ss}}$ & $1 / \wedge^{n}{ }^{n s}$ & $1 / \mathrm{N}^{\mathrm{ns}}$ & $1 / .^{\mathrm{ns}}$ & $1 / 9^{\text {ns }}$ & $1 / \Delta r^{\mathrm{ns}}$ & $9 \mathrm{D}$ \\
\hline 1 & $r / \varphi)^{*}$ & $r / q r^{\mathrm{ns}}$ & $r / q \gamma^{n s}$ & $r / T \gamma^{\mathrm{ns}}$ & $\mathrm{r} / \mathrm{s \Lambda} \mathrm{N}^{*}$ & $N 1^{* a}$ & $1 / r^{n s}$ & $N / 9 r^{\circ 8}$ & $1 / / v^{\mathrm{ns}}$ & $1 / N r^{n s}$ & $r / \cdot r^{\text {ns }}$ & $\kappa / F \cdot *$ & $r / \epsilon \Phi^{* * B}$ & $1 / 9 .{ }^{n s}$ & $F / r r^{*}$ & $1 / 1 q^{n s}$ & $1 / 99^{\text {ns }}$ & $r / 4)^{* \prime}$ & $r / N r^{*}$ & $r / \pi r^{\mathrm{ns}}$ & VD \\
\hline
\end{tabular}


جدول ^- مقايسه دو بدوى كروههاى كروموزومى براى واريانس درون كروموزومى صفت وزن هزار دانه با آزمون مدر شرايط بدون تنش (بالاى قطر) و در شرايط تنش (زير قطر) Ttable 8. Comparison of two primitive chromosome groups for intra-chromosomal variance of 1000 seed weight with f test under stressless conditions (above

\begin{tabular}{|c|c|c|c|c|c|c|c|c|c|c|c|c|c|c|c|c|c|c|c|c|c|}
\hline D & $9 \mathrm{D}$ & $\Delta \mathrm{D}$ & ${ }^{H} \mathrm{D}$ & rD & TD & ID & VB & $9 \mathrm{~B}$ & $\Delta \mathrm{B}$ & ${ }^{\prime} B$ & $\mu_{\mathrm{B}}$ & TB & IB & $\mathrm{VA}$ & $9 \mathrm{~A}$ & $\Delta \mathrm{A}$ & rA & rA & rA & IA & \\
\hline$\varphi / q^{*}$ & $r / \Lambda \Delta^{\mathrm{nS}}$ & $r / T Y^{n s}$ & $r / \& \varepsilon^{\mathrm{ns}}$ & $r / r^{n s}$ & $\mid r / \Delta^{* s}$ & $s / r^{* * *}$ & $r / \kappa^{n \text { ns }}$ & $\Delta / \cdot r^{*}$ & $r / \Delta f^{\mathrm{ns}}$ & $1 . / 199^{\circ 00}$ & $r / r \Lambda^{\mathrm{ns}}$ & $10 / N \Delta^{s s}$ & $r / 4 e^{\mathrm{ns}}$ & $s / \Delta V^{5 s}$ & $\Delta / T V^{*}$ & $r / A V^{*}$ & $r / T \gamma^{n s}$ & $r / \mathrm{VA}^{*}$ & $\mid c / 91^{\circ}$ & 1 & IA \\
\hline $1 / \cdot 9^{\text {ns }}$ & $1 / q \varphi^{\mathrm{ns}}$ & $r / \odot^{\mathrm{ns}}$ & $1 / 9 . \mathrm{ns}$ & $r / r^{\mathrm{ns}}$ & $r / q V^{\mathrm{ns}}$ & $1 / \Gamma^{\mathrm{ns}}$ & $1 / q r^{n s}$ & $1 / \cdot v^{\mathrm{ns}}$ & $1 / 4 e^{\mathrm{ns}}$ & $r / T e^{\mathrm{ns}}$ & $r / \cdot 0^{\mathrm{ns}}$ & 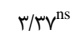 & $1 / \mathrm{vr}^{\mathrm{ns}}$ & $1 / \varphi^{\mathrm{ns}}$ & $1 / 1 r^{\text {ns }}$ & $1 / r 1^{\mathrm{ns}}$ & $1 / 4 r^{n s}$ & $1 / / \mathrm{F}^{\mathrm{nS}}$ & 1 & $1 / r \Lambda^{n s}$ & $r A$ \\
\hline $1 / / r^{\mathrm{ns}}$ & $1 / \% r^{n s}$ & $1 / 9 \mathrm{v}^{\mathrm{ns}}$ & $1 / \Delta f^{n s}$ & $1 / 9 \Delta^{\mathrm{nS}}$ & $r / r \cdot{ }^{\text {ns }}$ & $1 / 9 T^{\text {ns }}$ & $1 / \Delta S^{\mathrm{nS}}$ & $1 / \pi r^{\text {ns }}$ & $1 / \mathrm{q}^{\mathrm{ns}}$ & $r / w^{n s}$ & $1 / 99^{\mathrm{ns}}$ & $F / 18^{\circ}$ & $1 / 4 \varphi^{\mathrm{ns}}$ & $1 / \mathrm{Ne}^{\mathrm{ns}}$ & $1 / \% q^{\text {ns }}$ & $1 / \cdot r^{\mathrm{ns}}$ & $1 / 1 \varsigma^{\mathrm{ns}}$ & 1 & $r / r^{\text {ns }}$ & $r / q q^{n s}$ & ra \\
\hline $1 / \mu^{\mathrm{ns}}$ & $1 / 10^{\mathrm{ns}}$ & $1 / 4 \varphi^{\mathrm{ns}}$ & $1 / r^{\mathrm{ns}}$ & $1 / 19^{\mathrm{ns}}$ & $r / \Lambda r^{\text {ns }}$ & $1 / \mathrm{s}^{\mathrm{ns}}$ & $1 / \% \Delta^{\mathrm{ns}}$ & $1 / \Delta f^{\mathrm{ns}}$ & $1 / q^{\text {ns }}$ & $r / r^{\mathrm{ns}}$ & $1 / 4 r^{n s}$ & $r / \Lambda r^{* * *}$ & $1 / / \mathrm{r}^{\mathrm{ns}}$ & $r / 1^{\text {ns }}$ & $1 / 91^{\text {ns }}$ & $1 / \lambda^{\mathrm{ns}}$ & 1 & $1 / \varphi^{\mathrm{ns}}$ & $1 / 88^{\mathrm{ns}}$ & $r / / r^{\mathrm{ns}}$ & rA \\
\hline $1 / / 1^{\mathrm{ns}}$ & $1 / \% y^{\text {ns }}$ & $1 / \mathrm{v} 1^{\mathrm{ns}}$ & $1 / \Delta \mathrm{V}^{\mathrm{ns}}$ & $1 / 8 \Lambda^{\mathrm{ns}}$ & $r / r r^{\text {ns }}$ & $1 / \Delta \Lambda^{\mathrm{ns}}$ & $1 / 8 q^{n s}$ & $1 / r \cdot{ }^{\mathrm{ns}}$ & $1 / \Delta T^{n s}$ & $r / V^{\mathrm{ns}}$ & $1 / 99^{n s}$ & $\psi / \cdot V^{\circ}$ & $1 / \% \mathrm{v}^{\mathrm{ns}}$ & $1 / v^{n s}$ & $1 / \% \xi^{\mathrm{ns}}$ & 1 & $1 / \cdot r^{\mathrm{ns}}$ & $1 / \% \xi^{\text {ns }}$ & $1 / v^{n s}$ & $r / \Lambda^{\mathrm{ns}}$ & $\Delta \mathrm{A}$ \\
\hline $1 / \pi r^{\mathrm{ns}}$ & $1 / \Lambda \Delta^{\mathrm{nS}}$ & $r / r r^{n s}$ & $r / / e^{\mathrm{ns}}$ & $r / q^{\text {ns }}$ & $r / r v^{n s}$ & $1 / 18^{\mathrm{ns}}$ & $r / / V^{\mathrm{ns}}$ & $1 / \cdot 0^{\mathrm{ns}}$ & $r / \gamma^{\mathrm{ns}}$ & $1 / 99^{\text {ns }}$ & $r / \Psi^{\mathrm{ns}}$ & $r / 9 q^{\text {ns }}$ & $r / .^{\mathrm{ns}}$ & $1 / \pi \Delta^{\mathrm{ns}}$ & 1 & $r / r T^{\mathrm{ns}}$ & $r / r \omega^{\mathrm{nS}}$ & $r / / r^{\text {ns }}$ & $1 / \% \xi^{\mathrm{ns}}$ & $1 / \bullet^{\mathrm{ns}}$ & $\varphi_{\mathrm{A}}$ \\
\hline $1 / \Delta r^{n s}$ & $r / r)^{\text {ns }}$ & $r / q .{ }^{n s}$ & $r / q \gamma^{\mathrm{ns}}$ & $r / \mathcal{N}^{\mathrm{ns}}$ & $1 / 9 .{ }^{\text {ns }}$ & $1 / v^{\mathrm{ns}}$ & $r / Y I^{\text {ns }}$ & $1 / w^{\text {ns }}$ & $r / \Delta q^{\text {ns }}$ & $1 / 9 \cdot{ }^{\text {ns }}$ & $r / M^{n s}$ & $r / \kappa^{\mathrm{ns}}$ & $r / \& q^{\text {ns }}$ & 1 & $1 / r^{\mathrm{ns}}$ & $r / .^{\mathrm{ns}}$ & $r / q \tau^{n s}$ & $\% / \wedge^{\circ}$ & $1 / v \varsigma^{n s}$ & $1 / \Lambda^{\mathrm{ns}}$ & $\mathrm{VA}$ \\
\hline $1 / 9 r^{\mathrm{ns}}$ & $1 / \cdot \Lambda^{\mathrm{ns}}$ & $1 / 1 \gamma^{\mathrm{ns}}$ & $\mathrm{v} \cdot \mathrm{v}^{\mathrm{ns}}$ & $1 / 1 \omega^{\mathrm{ns}}$ & $F / N e^{*}$ & $r / \mu T^{n s}$ & $1 / \cdot 9^{\text {ns }}$ & $1 / 90^{\mathrm{ns}}$ & $1 / \cdot \kappa^{\mathrm{ns}}$ & $r / Q \Lambda^{\circ}$ & $1 / / \gamma^{\mathrm{ns}}$ & $\Delta / q V^{* * *}$ & 1 & $1 / \mu^{\mathrm{ns}}$ & $1 / 1 q^{\text {ns }}$ & $r / 14^{\circ}$ & $f / \varphi^{*}$ & $\Delta / \mathcal{F}^{200}$ & $r / f \varphi^{\mathrm{nS}}$ & 1/9. ${ }^{\mathrm{ss}}$ & $\mathrm{IB}$ \\
\hline$r / 9 Y^{*}$ & $\Delta / \Delta r^{* s}$ & $8 / 90^{\circ 8}$ & $s(9)^{*}$ & $8 / \Lambda \Delta^{\circ s}$ & $1 / r \varepsilon^{\mathrm{ns}}$ & $r / \Delta \mathrm{V}^{\mathrm{ns}}$ & $8 / 49^{* 0}$ & $1 / 1 r^{\mathrm{ns}}$ & $s / r . *$ & $1 / \Delta \cdot{ }^{\mathrm{ns}}$ & $8 / 9^{\circ \circ}$ & 1 & $N / T^{\text {of }}$ & $0 / 99^{50}$ & $r / q r^{\circ}$ & $r / .^{\mathrm{ns}}$ & $r / 0^{\mathrm{ns}}$ & $1 / \varepsilon \gamma^{\mathrm{ns}}$ & $r / f \cdot{ }^{\text {ns }}$ & $C / \pi \Delta^{\circ}$ & $\tau_{\mathrm{B}}$ \\
\hline $\mathrm{V} / \mathrm{M}^{\mathrm{ns}}$ & $1 / r \Delta^{\mathrm{ns}}$ & $1 / 0^{\mathrm{ns}}$ & $1 / \cdot \lambda^{\mathrm{ns}}$ & $1 / 1^{\mathrm{ns}}$ & $\Delta / \leftarrow \Lambda^{* * B}$ & $r / g \Lambda^{\text {ns }}$ & $1 / \cdot 8^{\mathrm{ns}}$ & $r / r 0^{\mathrm{ns}}$ & $\left(1 / 11^{\mathrm{ns}}\right.$ & $\mathrm{H} / \mathrm{G} 0^{\circ}$ & 1 & $r / \mu f^{\mathrm{ns}}$ & $r / \% \Lambda^{\text {ns }}$ & $1 / \Delta^{\mathrm{ns}}$ & $1 / r \Lambda^{\mathrm{ns}}$ & $1 / \mathrm{v}^{\mathrm{ns}}$ & $1 / q x^{n s}$ & $r / r \gamma^{\mathrm{ns}}$ & $1 / \cdot r^{\mathrm{ns}}$ & $1 / \Gamma 1^{\mathrm{ns}}$ & $r_{\mathrm{B}}$ \\
\hline$r / \& \Delta^{\mathrm{ns}}$ & $r / 89^{\circ}$ & $r / q r^{\circ}$ & $F / T V^{\circ}$ & $F / \Delta S^{\circ}$ & $1 / 19^{\text {ns }}$ & $1 / \mathrm{N}^{\mathrm{ns}}$ & $r / \pi T^{\circ}$ & $r / .9^{\text {ns }}$ & $r / r^{s}$ & 1 & $1 / 10^{\mathrm{ns}}$ & $r / 99^{\circ}$ & $r / \gamma^{\text {ns }}$ & $1 / q^{\mathrm{ns}^{\mathrm{s}}}$ & $1 / / \varphi^{\text {ns }}$ & $1 / \Delta r^{\text {ns }}$ & $1 / \mathrm{r} \mathrm{N}^{\mathrm{ns}}$ & $r / \& q^{n s}$ & $v / v^{\mathrm{ns}}$ & $1 / 19^{\mathrm{ns}}$ & ${ }_{\mathrm{B}} \mathrm{B}$ \\
\hline $1 / 9 q^{\text {ns }}$ & $1 / 1 r^{\mathrm{ns}}$ & $1 / 1 r^{\mathrm{ns}}$ & $1 / r^{\mathrm{ns}}$ & $1 / 1 \cdot^{\mathrm{ns}}$ & $r / Q T^{*}$ & $r / T^{\mathrm{ns}}$ & $1 / 0^{\mathrm{ns}}$ & $1 / \mathrm{A}^{\mathrm{ns}}$ & 1 & $1 / \mu e^{\mathrm{ns}}$ & $1 / / r^{n s}$ & $r / Q \Delta^{\mathrm{ns}}$ & $r / \Lambda)^{\text {ns }}$ & $r / r^{\mathrm{ns}}$ & $1 / \Delta 5^{n s}$ & $1 / \mathscr{E} \mathrm{v}^{\mathrm{ns}}$ & $1 / 4 \varphi^{\mathrm{ns}}$ & $r / l^{\mathrm{ns}}$ & $1 / 10^{\mathrm{ns}}$ & $1 / 4 \Lambda^{\mathrm{ns}}$ & $\mathrm{QB}$ \\
\hline $1 / / v^{\mathrm{ns}}$ & $1 / v q^{n s}$ & $r / \pi r^{n s}$ & $r / \kappa^{\mathrm{ns}}$ & $r / \Lambda^{\mathrm{ns}}$ & $r / q q^{n s}$ & $1 / \pi r^{\mathrm{ns}}$ & $\mathrm{r} / \mathrm{\gamma}^{\mathrm{ns}}$ & 1 & $1 / 99^{\mathrm{ns}}$ & $r / \Delta^{\mathrm{ns}}$ & $1 / \wedge \mathrm{n}^{\mathrm{ns}}$ & $1 / \mathrm{N}^{\mathrm{ns}}$ & $F / 90^{\circ}$ & $r / r^{\mathrm{ns}}$ & $r / \Delta q^{\text {ns }}$ & $1 / 1 r^{\mathrm{ns}}$ & $1 / 10^{\mathrm{ns}}$ & $(/ /)^{\mathrm{ns}}$ & $1 / 99^{\mathrm{ns}}$ & $r / \varphi^{\mathrm{ns}}$ & $\varepsilon_{\mathrm{B}}$ \\
\hline $\mathrm{I} / \mathrm{w} \mathrm{w}^{\mathrm{ns}}$ & $1 / / V^{\mathrm{ns}}$ & $1 / v^{\mathrm{ns}}$ & $1 / \cdot 1^{\mathrm{ns}}$ & $1 / 9^{\mathrm{ns}}$ & $\Delta / 1 Q^{*}$ & $r / \Delta \Gamma^{n s}$ & 1 & $1 / / \Lambda^{\mathrm{ns}}$ & $1 / 1^{\text {ns }}$ & $1 / \pi r^{n s}$ & $1 / / r^{n s}$ & $r / Q^{n s}$ & $r / V \Lambda^{n s}$ & $r / 1^{\mathrm{ns}}$ & $1 / \Delta \Delta^{\mathrm{nS}}$ & $1 / 4 q^{\text {ns }}$ & $1 / \kappa^{\mathrm{ns}}$ & $r / r^{\mathrm{ns}}$ & $1 / 1 e^{\mathrm{ns}}$ & $1 / \epsilon \epsilon^{\mathrm{ns}}$ & VB \\
\hline $1 / 4 r^{\text {ns }}$ & $r / 1 D^{\text {ns }}$ & $r / v{ }^{n s}$ & $r / F q^{n s}$ & $r / 9 q^{\text {ns }}$ & $r / \cdot e^{\text {ns }}$ & 1 & $r / r \Lambda^{\mathrm{ns}}$ & $1 / / \varphi^{\text {ns }}$ & $r / \tau \xi^{\text {ns }}$ & $r / \Lambda \cdot{ }^{\text {ns }}$ & $\mathrm{r} / \Delta \mathrm{O}^{\mathrm{ns}}$ & $1 / \mu^{\mathrm{ns}}$ & $s / 4 e^{a b s}$ & $F / \Delta \Lambda^{\circ}$ & $r / \Delta r^{*}$ & $1 / \Delta)^{n s}$ & $1 / \Delta \mathrm{V}^{\mathrm{ns}}$ & $1 / / r^{\mathrm{ns}}$ & $r / \varphi 0^{\text {ns }}$ & $r / \pi r^{n s}$ & ID \\
\hline$r / q)^{\mathrm{ns}}$ & $\mathrm{F} / \mathrm{rq}^{\circ}$ & $\Delta / \Delta \Delta^{20}$ & $\Delta / \cdot \Lambda^{*}$ & $\Delta / \psi^{* 60}$ & 1 & $1 / \Delta 5^{n s}$ & $1 / \kappa \xi^{\mathrm{ns}}$ & $1 / 1 e^{\mathrm{ns}}$ & $1 / \& \Delta^{\mathrm{ns}}$ & $1 / v q^{n s}$ & $1 / 9 y^{n s}$ & $r / \psi^{\mathrm{ns}}$ & $\% / \cdot v^{\circ}$ & $r / q \varphi^{\mathrm{ns}}$ & $r / r \gamma^{n s}$ & $1 / \cdot r^{\mathrm{ns}}$ & $1 / \cdot 1^{\mathrm{ns}}$ & $1 / \mathrm{rq}^{\mathrm{ns}}$ & $1 / 9 \mathrm{v}^{\mathrm{ns}}$ & $r / / r^{\mathrm{ns}}$ & TD \\
\hline$v / / \gamma^{\mathrm{ns}}$ & $1 / \pi e^{\mathrm{ns}}$ & $1 / \cdot \tau^{n s}$ & $v \cdot v^{\mathrm{ns}}$ & 1 & $1 / 9 .^{n s}$ & $r / q \vartheta^{n s}$ & $1 / r^{\mathrm{ns}}$ & $r / M \Lambda^{\mathrm{ns}}$ & $1 / / 1^{\text {ns }}$ & $1 / \bullet^{\mathrm{ns}}$ & $1 / 11^{\mathrm{ns}}$ & $r / A V^{\circ}$ & $r / / f^{\mathrm{ns}}$ & $1 / \Delta \Delta^{\mathrm{ns}}$ & $1 / 19^{\text {ns }}$ & $1 / 9 q^{n s}$ & $1 / 19^{\text {ns }}$ & $r / q \psi^{\mathrm{ns}}$ & $1 / 1 f^{\mathrm{ns}}$ & $1 / / \pi^{n s}$ & $r_{\mathrm{D}}$ \\
\hline $1 / v \Delta^{\mathrm{ns}}$ & $1 / 1 \gamma^{\mathrm{ns}}$ & $1 / 9^{\text {ns }}$ & 1 & $1 / 99^{\text {ns }}$ & $1 / \cdot 0^{\mathrm{ns}}$ & $1 / 4 q^{\text {ns }}$ & $1 / \Delta r^{n s}$ & $1 / \cdot 9^{\mathrm{ns}}$ & $1 / \Delta 1^{\text {ns }}$ & $v / / v^{n s}$ & $1 / \mathrm{v}^{\mathrm{ns}}$ & $1 / 9 \Delta^{\mathrm{ns}}$ & $F / T D^{\circ}$ & $r / \gamma^{\mathrm{ns}}$ & $r / \% \gamma^{n s}$ & $1 / \cdot r^{\mathrm{ns}}$ & $1 / 0^{\mathrm{ns}}$ & $1 / r r^{n s}$ & $1 / v Q^{\mathrm{nS}}$ & $r / r r^{n s}$ & ${ }^{*} \mathrm{D}$ \\
\hline $1 / 19^{\text {ns }}$ & $1 / r r^{\mathrm{ns}}$ & 1 & $s / \psi \gamma^{* * B}$ & $r / r)^{\text {ns }}$ & $8 / 1 . *$ & $\checkmark / \Delta \omega^{* * *}$ & $4 / 19^{\circ}$ & $8 / 91^{5 *}$ & $F / T^{\circ}$ & $r / \mu 0^{*}$ & $r / r r^{2}$ & $\mid T / F T^{\circ B}$ & $1 / \Delta .^{\text {ns }}$ & $r / \gamma^{\mathrm{ns}}$ & $r / q q^{\text {ns }}$ & $g / T T^{\circ B}$ & $91.0^{* 88}$ & $N / F D^{* 0}$ & $r / 90^{\circ}$ & $r / \Lambda \Delta^{\mathrm{ns}}$ & $\Delta \mathrm{D}$ \\
\hline $1 / \Delta)^{\mathrm{ns}}$ & 1 & $g / \pi)^{\circ e}$ & $1 / \cdot r^{\text {ns }}$ & $1 / q r^{\text {ns }}$ & $1 / r^{\mathrm{ns}}$ & $1 / \Delta r^{n s}$ & $1 / \& q^{\text {ns }}$ & $1 / 1 r^{\mathrm{ns}}$ & $1 / \varepsilon \Lambda^{\text {ns }}$ & $\sqrt{1 / \mu r^{\mathrm{ns}}}$ & $1 / 9 \mathrm{y}^{\mathrm{ns}}$ & $r / .^{\mathrm{ns}}$ & $r / F^{\circ}$ & $r / .^{\mathrm{ns}}$ & $r / T)^{\text {ns }}$ & $1 / .^{\mathrm{ns}}$ & $1 / \cdot r^{\mathrm{ns}}$ & $1 / \Psi \varepsilon^{n s}$ & $\mathrm{~V} / \mathrm{v} \cdot{ }^{\mathrm{ns}}$ & $r / \backslash \Lambda^{\mathrm{ns}}$ & $\Leftrightarrow \mathrm{D}$ \\
\hline 1 & $\kappa / 4 \Lambda^{\circ}$ & $1 / 4 r^{n s}$ & $r / \Delta \cdot{ }^{\mathrm{ns}}$ & $r / r q^{n s}$ & $+/ q^{*}$ & g/v."*t & $r / q r^{n S}$ & $r / q T^{\circ}$ & $r / q \gamma^{n s}$ & $r / s \cdot$ ns & $r / q \Psi^{n s}$ & $N N \mathcal{G}^{* * *}$ & $1 \cdot \bullet^{\mathrm{ns}}$ & $1 / k \xi^{\mathrm{ns}}$ & $1 / q^{\text {ns }}$ & $r / T V^{*}$ & $F / T V^{\circ}$ & $\Delta / Q Q^{* 6}$ & $r / \Delta \Lambda^{\mathrm{ns}}$ & $\mathrm{r} / 0^{\mathrm{ns}}$ & VD \\
\hline
\end{tabular}


جدول ج- مقايسه دو بدوى كروههاى كروموزومى براى واريانس درون كروموزومى صفت شاخص برداشت (بالاى قطر) و تعداد دانه در سنبله (يايين قطر) در شرايط تنش Table 9. Comparison of two primitive chromosome groups for in-chromosomal variance, harvest index (above diameter) and number of seeds per spike (lower diameter) under

\begin{tabular}{|c|c|c|c|c|c|c|c|c|c|c|c|c|c|c|c|c|c|c|c|c|c|}
\hline VD & $9 \mathrm{D}$ & $\Delta \mathrm{D}$ & ${ }^{k} \mathrm{D}$ & $r \mathrm{D}$ & rD & ID & VB & $9 \mathrm{~B}$ & $\Delta \mathrm{B}$ & ${ }^{\mu} \mathrm{B}$ & $\mu_{\mathrm{B}}$ & rB & IB & VA & $9 \mathrm{~A}$ & $\Delta \mathrm{A}$ & $r_{\mathrm{A}}$ & ra & rA & $\mathrm{IA}$ & \\
\hline$r / \Delta \Lambda^{\mathrm{nS}}$ & $1 / \Delta \cdot{ }^{n s}$ & $1 / 1 \mathrm{n}^{\mathrm{ns}}$ & $r / \Gamma)^{\mathrm{nS}}$ & $1 / 11^{\text {ns }}$ & $r / / r^{\mathrm{ns}}$ & $|/ /|^{\mathrm{ns}}$ & $1 / M^{n s}$ & $1 / r^{n{ }^{n s}}$ & $V / \Delta 1^{n s}$ & $1 / \mathrm{rq}^{\mathrm{ns}}$ & $r / 9 \Lambda^{*}$ & $1 Q / \Delta V^{* \theta}$ & $1 / 9 V^{\mathrm{ns}}$ & $|/ / 9|^{\mathrm{ns}}$ & $1 / 9 \cdot{ }^{\mathrm{ns}}$ & $1 / r \mathrm{rss}^{\mathrm{nS}}$ & $r / \cdot 1^{\mathrm{ns}}$ & $1 / 9 T^{1 \mathrm{~ns}}$ & $\sqrt{1 / q^{n S s}}$ & & $\mathrm{IA}$ \\
\hline $1 / \Delta q^{\mathrm{ns}}$ & $1 / \cdot 9^{\text {ns }}$ & $1 / 4 r^{n s}$ & $1 / 4 \gamma^{n s}$ & $1 / \notin \varepsilon^{\mathrm{ns}}$ & $1 / 4 r^{\mathrm{ns}}$ & $1 / 11^{\mathrm{ns}}$ & $r / \bullet^{\mathrm{ns}}$ & $1 / \sim^{\mathrm{ns}}$ & $1 / \mathrm{r}^{\mathrm{ns}}$ & $r / q^{n s}$ & $r / r^{\mathrm{ns}}$ & $\checkmark / \Delta V^{20}$ & $1 / \cdot r^{\mathrm{ns}}$ & $r / q r^{n s}$ & $1 / \cdot r^{n s}$ & $r / r^{\mathrm{ns}}$ & $1 / r^{\text {ns }}$ & $1 / / \Lambda^{\mathrm{ns}}$ & & $1 / q T^{n s}$ & rA \\
\hline $1 / / \psi^{\mathrm{ns}}$ & $1 / r \Lambda^{n s}$ & $1 / 9 \mathrm{r}^{\mathrm{ns}}$ & $1 / 19^{\mathrm{ns}}$ & $1 / r^{n s}$ & $1 / r^{\mathrm{ns}}$ & $1 / \cdot 9^{\mathrm{ns}}$ & $r|q|^{*}$ & $1 / 9 .{ }^{n s}$ & $1 / r r^{n s}$ & $r / \notin V^{\mathrm{ns}}$ & $1 / 9 r^{\mathrm{ns}}$ & $N M$ & $1 / 1 Q^{\mathrm{ns}}$ & $r / . q^{\mathrm{ns}}$ & $1 / r^{n s}$ & $\Gamma / \sim^{n n^{n s}}$ & $1 / f^{\mathrm{ns}}$ & & $1 / \kappa^{n s}$ & $1 / 1 \mathrm{f}^{\mathrm{ns}}$ & rA \\
\hline $1 / q^{\text {ns }}$ & $1 / r y^{n s}$ & $1 / v^{\mathrm{ens}}$ & $1 / / r^{\mathrm{ns}}$ & $1 / \mathrm{n}^{\mathrm{ns}}$ & $1 / 19^{\mathrm{ns}}$ & $1 / 1^{\mathrm{ns}}$ & $r / V^{*}$ & $1 / 9 \mathrm{~V}^{\mathrm{ns}}$ & $1 / r^{n s}$ & $r / \Delta \Lambda^{\mathrm{ns}}$ & $1 / \wedge n^{\mathrm{ns}}$ & $V / W^{* s}$ & $1 / r^{n s}$ & $r / r r^{\mathrm{ns}}$ & $1 / r \omega^{\mathrm{ns}}$ & $F /\left.\epsilon^{n}\right|^{\text {ns }}$ & & $v / \Delta \gamma^{n s}$ & $1 / 1^{\mathrm{ns}}$ & $1 / 1 .^{\text {ns }}$ & ra \\
\hline$r / r .{ }^{\text {ns }}$ & $1 / \Lambda \Delta^{\mathrm{ns}}$ & $1 / 4 r^{n s}$ & $r / W^{\text {ns }}$ & $1 / r^{n s}$ & $r / A r^{\mathrm{ns}}$ & $T / r^{\mathrm{ns}}$ & $1 / \Delta n^{n s}$ & $1 / 4 q^{\text {ns }}$ & $1 / M^{n s}$ & $1 / \cdot f^{\mathrm{ns}}$ & $r / \Delta \phi^{*}$ & $19 / \mu \cdot * *$ & $r / \cdot r^{n s}$ & $1 /{ }^{n}{ }^{\mathrm{ns}}$ & $1 / 94^{\mathrm{ns}}$ & & $1 / \wedge^{n}{ }^{\mathrm{ns}}$ & $1 / 10^{\mathrm{ns}}$ & $1 / q r^{n s}$ & $1 / .^{\mathrm{ns}}$ & $\triangle \mathrm{A}$ \\
\hline $1 / 9)^{\text {ns }}$ & $1 / \cdot v^{n s}$ & $1 / \mathrm{rq}^{\mathrm{ns}}$ & $1 / 4 .{ }^{\text {ns }}$ & $1 / \varphi^{\mathrm{ns}}$ & $1 / \% \Delta^{\mathrm{ns}}$ & $1 / 1 r^{n s}$ & $r / 1^{\text {ns }}$ & $1 / r^{n s}$ & $1 / \cdot 0^{\mathrm{ns}}$ & $r / \cdot \cdot^{\mathrm{ns}}$ & $r / \mu \cdot{ }^{n s}$ & $\checkmark / r^{* 8 s}$ & $1 / \cdot e^{\text {ns }}$ & $r / \Delta r^{\text {ns }}$ & & $1 / \Delta r^{n s}$ & $r / \Lambda r^{\text {ns }}$ & $1 / \wedge \cdot{ }^{\text {ns }}$ & $r /\left.\Delta\right|^{n s}$ & $1 / \Delta \mathrm{n}^{\mathrm{ns}}$ & $9 \mathrm{~A}$ \\
\hline$F / 10^{\circ}$ & $r / \epsilon^{n s}$ & $1 / \wedge \Delta^{\mathrm{ns}}$ & $r / 8 .{ }^{\circ}$ & $1 / v^{n s}$ & $r / N r^{*}$ & $r / q .{ }^{n s}$ & $1 / / v^{n s}$ & $1 / 9 n^{\text {ns }}$ & $r / 4 t^{\mathrm{ns}}$ & $1 / r \omega^{\mathrm{ns}}$ & $\Delta / q r^{* s}$ & $r \Delta / \cdot \varphi^{* * *}$ & $r / \wedge^{\mathrm{ns}}$ & & $1 / / \mathrm{f}^{\mathrm{ns}}$ & $1 / \sim r^{n s}$ & $r / \kappa \Lambda^{\mathrm{ns}}$ & $1 / \Delta \gamma^{\mathrm{ns}}$ & $r / r r^{n s}$ & $1 / r^{\mathrm{ns}}$ & VA \\
\hline $1 / \Delta \Delta^{\text {ns }}$ & $1 / 1^{\mathrm{ns}}$ & $1 / e^{\mathrm{nS}}$ & $1 / r r^{n s}$ & $1 / \Delta \cdot{ }^{n s}$ & $1 / \mathrm{ma}^{\mathrm{ns}}$ & $1 / \cdot \wedge^{\mathrm{ns}}$ & $r / l e^{\mathrm{ns}}$ & $1 / \mathrm{rq}^{\mathrm{ns}}$ & $1 / 1 .^{n s}$ & $r / l \mathrm{~F}^{\mathrm{ns}}$ & $r / r)^{\mathrm{ns}}$ & $q / r Y^{\infty s}$ & & $r / T^{\circ}$ & $r / \Lambda r^{\prime \prime}$ & $r / \cdot v^{\text {ns }}$ & $1 / v^{n s}$ & $r / 8 \Lambda^{\text {ns }}$ & $1 / 9 .{ }^{n s}$ & $r / \cdot v^{n s}$ & IB \\
\hline $91 \cdot r^{\circ 8}$ & $1 . / 4 r^{* * *}$ & $\mid \Psi / \Delta \omega^{* \Delta}$ & $8 / 99^{* \Delta s}$ & $1 \% / Q \Lambda^{* s}$ & $s(N)^{* *}$ & $N / \mathbb{Y}^{* 8 s}$ & $r q / r . * *$ & $T r / Q \Delta^{\circ \theta}$ & $1 . / \pi e^{* s}$ & $r .1 . r^{* * *}$ & $r / \mu^{*}$ & & $r / 1^{n s}$ & $1 / r^{\mathrm{ns}}$ & $1 / \Delta \Delta^{\mathrm{ns}}$ & $1 / \cdot 1^{\mathrm{ns}}$ & $1 / \wedge r^{\mathrm{ns}}$ & $1 / 19^{\mathrm{ns}}$ & $1 / 94^{\mathrm{ns}}$ & $1 / \cdot 1^{\mathrm{ns}}$ & rB \\
\hline $1 / 4 r^{\mathrm{ns}}$ & $r / \epsilon \xi^{\mathrm{ns}}$ & $r / r .{ }^{n s}$ & $1 / \varphi^{\mathrm{ns}}$ & $r / r .{ }^{n s}$ & $1 / \Delta q^{n s}$ & $r / \cdot r^{\mathrm{ns}}$ & $8 / 9 \Upsilon^{5 s}$ & $r / \cdot \bullet^{\mathrm{ns}}$ & $r / 4 r^{n s}$ & $r / r^{*}$ & & $1 / 19^{n s}$ & $r / v)^{*}$ & $1 / v^{\mathrm{ens}}$ & $1 / \mathrm{rn}^{\mathrm{ns}}$ & $1 / T^{\mathrm{ns}}$ & $r / \Lambda^{\mathrm{ns}}$ & $1 / \mu^{\mathrm{ns}}$ & $1 / 9 q^{n s}$ & $1 / r 1^{\mathrm{ns}}$ & ${ }^{\mathrm{B}}$ \\
\hline$r / r r^{\text {ns }}$ & $1 / 9 r^{n s}$ & $1 / \kappa^{\mathrm{ns}} \mathrm{n}^{\mathrm{ns}}$ & $r / M^{\mathrm{ns}}$ & $1 / 4 \mathrm{rns}^{\mathrm{ns}}$ & $r / \Lambda^{\mathrm{ns}}$ & $r / r^{n s}$ & $1 / \epsilon^{\mathrm{ns}}$ & $1 / \Delta \Delta^{\mathrm{ns}}$ & $1 / 9 \Delta^{\mathrm{ns}}$ & & $\gamma / .^{\mathrm{ns}}$ & $1 / 9 \AA^{\mathrm{ns}}$ & $1 / \wedge \Delta^{\mathrm{ns}}$ & $r / r \Lambda^{\mathrm{ns}}$ & $r / q)^{\mathrm{ns}}$ & $1 / 99^{\mathrm{ns}}$ & $1 / \cdot^{\mathrm{ns}}$ & $1 / \kappa^{\mathrm{ns}}$ & $1 / \cdot r^{\mathrm{ns}}$ & $1 / 99^{\mathrm{ns}}$ & ${ }^{\mu} \mathrm{B}$ \\
\hline $1 / v \cdot{ }^{n s}$ & $1 / \cdot r^{\mathrm{ns}}$ & $1 / r^{n s}$ & $1 / \mathrm{F}^{\mathrm{ns}}$ & $1 / r^{\mathrm{ns}}$ & $1 / \Delta r^{\mathrm{ns}}$ & $1 / 19^{n s}$ & $r / N^{\mathrm{ns}}$ & $1 / r \mathrm{n}^{\mathrm{ns}}$ & & $r / q .{ }^{n s}$ & $1 /{ }^{n . n s}$ & $1 / \Delta \Delta^{\mathrm{ns}}$ & $F|\Lambda|^{*}$ & $1 / / \mathrm{n}^{\mathrm{ns}}$ & $1 / .^{\mathrm{ns}}$ & $1 / \Delta \mathrm{n}^{\mathrm{ns}}$ & $r / \Lambda r^{\mathrm{ns}}$ & $1 / v^{n s}$ & $r /\left.\Delta\right|^{\mathrm{ns}}$ & $1 / \Delta \mathrm{n}^{\mathrm{ns}}$ & $\triangle \mathrm{B}$ \\
\hline$r / 1 Q^{\text {ns }}$ & $1 / r e^{n s}$ & $1 / \cdot 0^{\mathrm{ns}}$ & $1 / \wedge^{n s}$ & $1 / \cdot n^{\mathrm{ns}}$ & $1 / 9 r^{n s}$ & $1 / \Delta \cdot{ }^{n s}$ & $r / r^{n s}$ & & $1 / 9^{\text {ns }}$ & $r / r^{n{ }^{n s}}$ & $1 / 19^{n s}$ & $1 / \varepsilon r^{n s}$ & $r / \kappa$, & $1 / 0^{\mathrm{ns}}$ & $1 / 9^{\text {ns }}$ & $1 / 4 e^{n s}$ & $r / 4 .{ }^{\mathrm{ns}}$ & $1 / 9 \Delta^{\mathrm{ns}}$ & $r / \mu^{n \mathrm{~ns}}$ & $1 / \kappa^{e n s}$ & $q_{\mathrm{B}}$ \\
\hline$r / N^{*}$ & $r / \Lambda)^{n s}$ & $r / \backslash \gamma^{n s}$ & $F / T^{*}$ & $r / 1 .{ }^{n s}$ & $r / \Psi^{\circ}$ & $r / 4 \cdot{ }^{n s}$ & & $1 / r \cdot{ }^{n s}$ & $1 / 41^{n s}$ & $1 / \wedge f^{\mathrm{ns}}$ & $1 / 9^{\text {ns }}$ & $1 / 1 .^{n s}$ & $r / \uparrow^{n s}$ & $1 / r \kappa^{\mathrm{ns}}$ & $1 / \kappa^{n \mathrm{~ns}}$ & $1 / 1^{\mathrm{ns}}$ & $r / .{ }^{\text {ns }}$ & $1 / r r^{n s}$ & $1 /{ }^{\mathrm{ns}}$ & $1 / 1^{\mathrm{ns}}$ & VB \\
\hline $1 / \kappa n^{n s}$ & $1 / r 1^{\mathrm{ns}}$ & $1 / \Delta \mathrm{n}^{\mathrm{ns}}$ & $1 / r \mathrm{ens}^{\mathrm{ns}}$ & $1 / q^{n n s}$ & $1 / r \Lambda^{\mathrm{ns}}$ & & $\Delta / r^{-s}$ & s/va & $\mathrm{V} / \mathrm{TV}^{* * *}$ & $r / \wedge r^{\mathrm{ns}}$ & 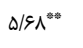 & $F / V \varphi^{*}$ & $1 / \Delta r^{n s}$ & $\varepsilon / \mathbb{F}^{* * \Delta}$ & $V / r^{* * s}$ & $F / N^{\circ}$ & $r / \varphi^{\text {ns }}$ & $4 M^{*}$ & $r / q)^{n s}$ & $r / v \cdot *$ & ID \\
\hline $1 / 1^{\text {ns }}$ & $1 / \Delta \Delta^{\mathrm{nS}}$ & $r / \cdot r^{n s}$ & $1 / \cdot e^{\mathrm{ns}}$ & $r / \cdot \wedge^{\mathrm{ns}}$ & & $\mathrm{V} / \cdot \mathrm{1}^{\circ \mathrm{s}}$ & $1 / r^{n s}$ & $1 / \cdot r^{n s}$ & $1 / \cdot 0^{\mathrm{ns}}$ & $r / \Psi^{\mathrm{ns}}$ & $1 / r^{r^{n s}}$ & $1 / \mathbb{e r}^{\mathrm{ns}}$ & $r / \Delta V^{*}$ & $1 / \cdot n^{\mathrm{ns}}$ & $1 / \cdot 0^{\mathrm{ns}}$ & $1 / \kappa^{n \mathrm{~ns}}$ & $r / q \Lambda^{\mathrm{ns}}$ & $1 / v \cdot{ }^{n s}$ & $r / 4)^{\mathrm{ns}}$ & $1 / \leftarrow^{n \mathrm{~ns}}$ & TD \\
\hline$r / r r^{\mathrm{ns}}$ & $1 / \% \varphi^{n s}$ & $1 / \cdot r^{\mathrm{ns}}$ & $r / \cdot)^{\mathrm{ns}}$ & & $F / F^{\circ}$ & $1 / 99^{n s}$ & $r / \cdot \wedge^{\mathrm{ns}}$ & $4 / 1^{\circ}$ & $F / \widetilde{r} \Delta^{\circ}$ & $r / \psi^{\text {ns }}$ & $r / \Psi^{\text {ns }}$ & $r / \Lambda)^{n s}$ & $1 / 1^{\mathrm{ns}}$ & $r / A r^{*}$ & $\varepsilon / \Psi^{*}$ & $r / V \Lambda^{n s}$ & $1 / \Delta f^{\mathrm{ns}}$ & $r / F r^{n s}$ & $1 / v^{n s}$ & $r / V^{n s}$ & $r_{\mathrm{D}}$ \\
\hline $1 / 10^{\mathrm{ns}}$ & $1 / \Delta \cdot{ }^{n s}$ & $1 / 9 \Delta^{\mathrm{ns}}$ & & $r / \Delta r^{*}$ & $1 / 1 v^{\mathrm{ns}}$ & $\Delta / q v^{\circ s}$ & $1 / 1 f^{\mathrm{ns}}$ & $1 / 1 f^{\mathrm{ns}}$ & $1 / \pi e^{\mathrm{ns}}$ & $1 / 9 \mathrm{~V}^{\mathrm{ns}}$ & $1 / \cdot 0^{\mathrm{ns}}$ & $1 / r \omega^{\mathrm{ns}}$ & $r / \wedge q^{*}$ & $1 / 9^{\text {ns }}$ & $1 / \pi \mu^{\mathrm{ns}}$ & $1 / T r^{n s}$ & $r / \Gamma \Lambda^{\mathrm{ns}}$ & $1 / \kappa^{\mathrm{sis}}$ & $r / \cdot \omega^{\mathrm{ns}}$ & $1 / T v^{n s}$ & ${ }^{4} \mathrm{D}$ \\
\hline$r / r \Delta^{\mathrm{ns}}$ & $1 / r^{n s}$ & & $1 / 9 \mathrm{n}^{\mathrm{ns}}$ & $8 / \Delta Q^{* * \Delta}$ & $1 / 8 \Lambda^{\mathrm{ns}}$ & $11 / \gamma^{* w}$ & $r / r \Delta^{\mathrm{ns}}$ & $1 / v r^{n s}$ & $1 / 4 \cdot{ }^{\mathrm{ns}}$ & $r / 1 .^{\text {ns }}$ & $r / \cdot v^{\mathrm{ns}}$ & $r / \notin v^{n s}$ & $V / 9 \Lambda^{* a s}$ & $1 / \wedge r^{n s}$ & $1 / \Delta Q^{n s}$ & $r / \Delta \cdot{ }^{n s}$ & $F(\Delta)^{\circ}$ & $r / \mathcal{N}^{\mathrm{ns}}$ & $\varphi / .0^{\circ}$ & $r / \Delta \cdot{ }^{n s}$ & $\Delta \mathrm{D}$ \\
\hline $1 / w^{n s}$ & & $\Delta / R \varphi^{\circ}$ & $r / q \gamma^{\mathrm{ns}}$ & $1 / r r^{n s}$ & $\left.r / \psi^{n}\right)^{\mathrm{ns}}$ & $T / \psi^{\mathrm{ns}}$ & $r / r^{\text {ns }}$ & $r / \cdot r^{\text {ns }}$ & $r / r^{q^{n s}}$ & $F / 10^{* 0}$ & $T / \Delta T^{\text {ns }}$ & $r / r^{n s}$ & $1 / \epsilon^{\mathrm{ns}}$ & $r / \wedge^{n s}$ & $r / r^{n s}$ & $r / 1 .{ }^{n s}$ & $1 / / v^{\text {ns }}$ & $1 / \wedge e^{\mathrm{ns}}$ & $1 / r r^{n s}$ & $r / 1 .{ }^{n s}$ & $9 \mathrm{D}$ \\
\hline & $1 / \cdot \Lambda^{\mathrm{ns}}$ & $\Delta / . \odot^{\theta *}$ & $r / \wedge r^{\mathrm{ns}}$ & $1 / r^{r^{n s}}$ & $r / r v^{\mathrm{ns}}$ & $r / \cdot \Lambda^{\text {ns }}$ & $r(\Delta)^{\mathrm{ns}}$ & $r / T v^{n s}$ & $\Gamma / \Delta \Delta^{\circ}$ & $1 / T r^{\mathrm{ns}}$ & $r / N^{\text {ns }}$ & $r / r q^{n s}$ & $1 /\left.9\right|^{\mathrm{ns}}$ & $r / 1^{\mathrm{ns}}$ & $r / \Delta \phi^{*}$ & $r / T r^{n s}$ & $1 / r^{\text {ns }}$ & $1 / 91^{\mathrm{ns}}$ & $1 / \mathrm{n}^{\mathrm{ns}}$ & $r / r Y^{n s}$ & VD \\
\hline
\end{tabular}



بررسى تنوع درون كروموزومى و بين كروموزومهاى هميولوگ براى عملكرد و اجزاى آن با استفاده از .

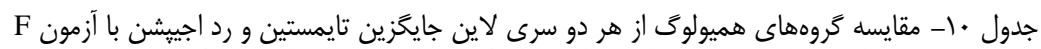
Table 10. Comparison of homologous groups from substitution lines of Timstein and Red Egyptian' with F-test

\begin{tabular}{|c|c|c|c|}
\hline شاخص برداشت & وزن هزار دانه (كرم) & عملكرد دانه (كرم) & كروههاى هميولوى \\
\hline تنش & بدون تنش & تنش & \\
\hline 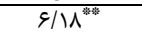 & $r / v r^{2}$ & $9 / 4 V^{(2 \pi / 9}$ & A1B1D1, A2B2D2 \\
\hline$r / 4 \Lambda^{*}$ & $1 / \cdot 1^{\mathrm{ns}}$ & 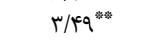 & $\mathrm{A} 1 \mathrm{~B} 1 \mathrm{D} 1, \mathrm{~A} 3 \mathrm{~B} 3 \mathrm{D} 3$ \\
\hline $1 / 4 \Lambda^{\mathrm{ns}}$ & $1 / M^{n s}$ & $r / 4 \Lambda^{*}$ & A1B1D1, A4B4D4 \\
\hline $1 / 91^{\mathrm{ns}}$ & $1 / r^{n s}$ & $r / \cdot \cdot^{\mathrm{ns}}$ & A1B1D1, A5B5D5 \\
\hline $1 / 1 .{ }^{\mathrm{ns}}$ & $1 / r^{n s}$ & $1 / .^{n s}$ & A1B1D1, A6B6D6 \\
\hline $1 / 9^{\mathrm{ns}}$ & $1 / 9 \Lambda^{\mathrm{ns}}$ & $r / q Y^{*}$ & A1B1D1, A7B7D7 \\
\hline$r / \& q^{*}$ & $F / . . *$ & $1 / \mathcal{}^{\mathrm{ns}}$ & $\mathrm{A} 2 \mathrm{~B} 2 \mathrm{D} 2, \mathrm{~A} 3 \mathrm{~B} 3 \mathrm{D} 3$ \\
\hline $11 / \Gamma \Delta^{\text {was }}$ & $1 / 9 \Lambda^{\mathrm{ns}}$ & $r|s|^{*}$ & $\mathrm{~A} 2 \mathrm{~B} 2 \mathrm{D} 2, \mathrm{~A} 4 \mathrm{~B} 4 \mathrm{D} 4$ \\
\hline १/৭४"*w & $r / 9 e^{m-w}$ & $r / \Lambda Q^{\text {stat }}$ & $\mathrm{A} 2 \mathrm{~B} 2 \mathrm{D} 2, \mathrm{~A} 5 \mathrm{~B} 5 \mathrm{D} 5$ \\
\hline 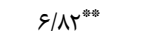 & $r / A V^{*}$ & 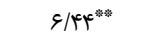 & $\mathrm{A} 2 \mathrm{~B} 2 \mathrm{D} 2, \mathrm{~A} 6 \mathrm{~B} 6 \mathrm{D} 6$ \\
\hline$g / N)^{* w a s}$ & $r / r)^{\mathrm{ns}}$ & $r / r r^{\mathrm{ns}}$ & $\mathrm{A} 2 \mathrm{~B} 2 \mathrm{D} 2, \mathrm{~A} 7 \mathrm{~B} 7 \mathrm{D} 7$ \\
\hline$r / \Delta \Delta^{* * *}$ & $r / \cdot r^{\mathrm{ns}}$ & $\mid / 41^{\mathrm{ns}}$ & A3B3D3, A4B4D4 \\
\hline$\varphi / .$. *t: & $1 / r^{\mathrm{ns}}$ & $1 / 99^{n s}$ & A3B3D3, A5B5D5 \\
\hline$T / V e^{*}$ & $1 / q^{n s}$ & 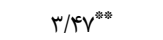 & $\mathrm{A} 3 \mathrm{~B} 3 \mathrm{D} 3, \mathrm{~A} 6 \mathrm{~B} 6 \mathrm{D} 6$ \\
\hline$r / 9 q^{*}$ & $\mid /(\Lambda)^{\mathrm{ns}}$ & $1 / 19^{\mathrm{ns}}$ & A3B3D3, A7B7D7 \\
\hline $1 / / f^{\mathrm{ns}}$ & $r / \& q^{*}$ & $1 / r^{\mathrm{ns}}$ & A4B4D4, A5B5D5 \\
\hline $1 / 98^{\mathrm{ns}}$ & $1 / F \Delta^{\mathrm{ns}}$ & $r / \& q^{*}$ & $\mathrm{~A} 4 \mathrm{~B} 4 \mathrm{D} 4, \mathrm{~A} 6 \mathrm{~B} 6 \mathrm{D} 6$ \\
\hline $1 / q^{\mathrm{ns}}$ & $1 / 1 r^{\mathrm{ns}}$ & $\backslash / \Lambda^{\mathrm{ns}}$ & $\mathrm{A} 4 \mathrm{~B} 4 \mathrm{D} 4, \mathrm{~A} 7 \mathrm{~B} 7 \mathrm{D} 7$ \\
\hline $1 / 49^{\mathrm{ns}}$ & $1 / N r^{n s}$ & $r / \Delta^{\mathrm{ns}}$ & A5B5D5, A6B6D6 \\
\hline $1 / \leftarrow \Lambda^{\mathrm{ns}}$ & $r / \pi^{n s}$ & $1 / \& r^{\mathrm{ns}}$ & A5B5D5, A7B7D7 \\
\hline $1 / \cdot r^{\mathrm{ns}}$ & $1 / \mu \cdot{ }^{\mathrm{ns}}$ & $r / q . *$ & A6B6D6, A7B7D7 \\
\hline
\end{tabular}

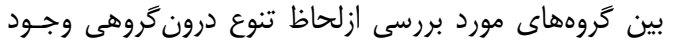

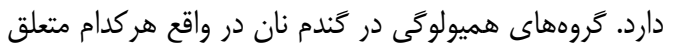

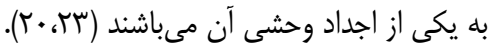

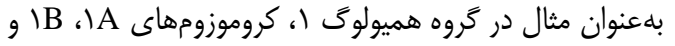

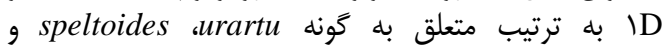
tauschii

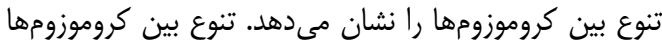

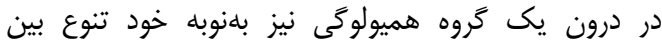

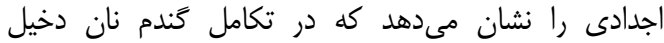

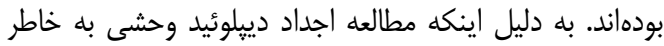

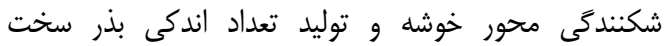

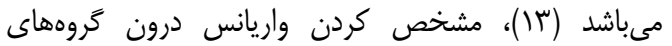

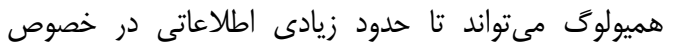

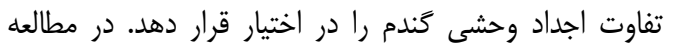

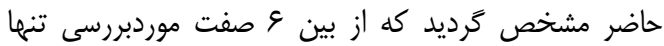

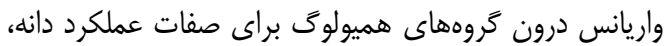

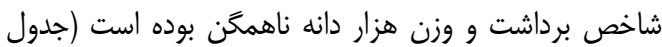

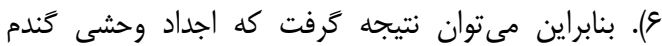

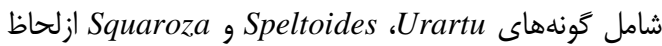
اين صفات متفاوت و ازلحاظ ب صفت ديخر يكسان بودهان انداند.

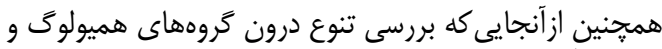

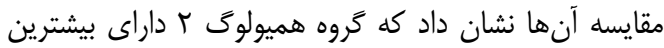

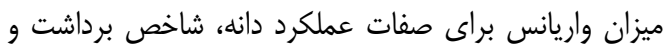

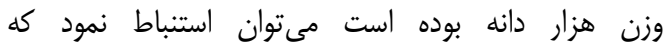

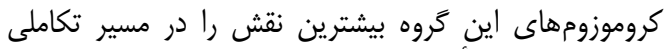

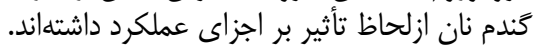

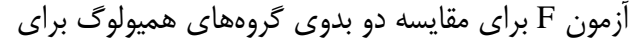

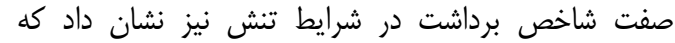

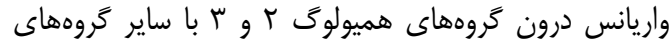

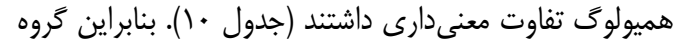

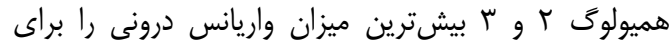

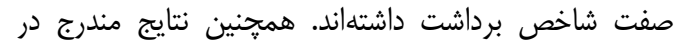

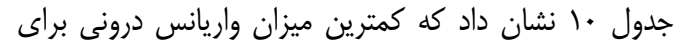

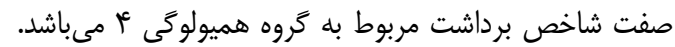

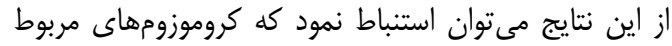

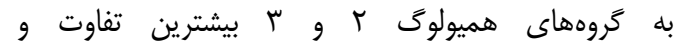

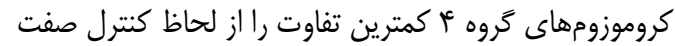
شاخص برداشت با همديخر داشتهاند.

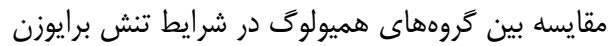

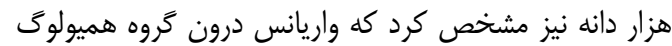

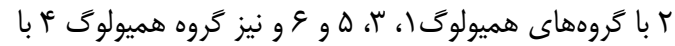

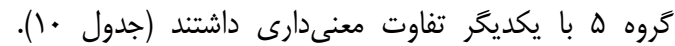

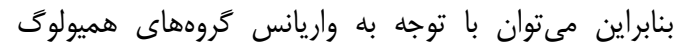

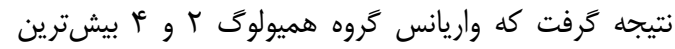

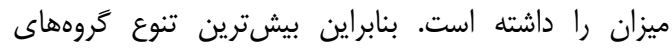

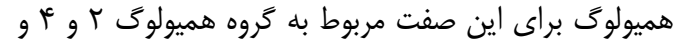

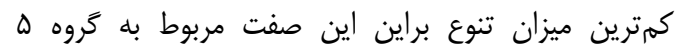
مىباشد. از نتايج فوق مىتوان استنباط نمود كه از بين گروههاى

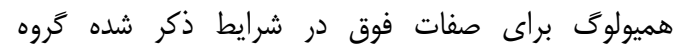

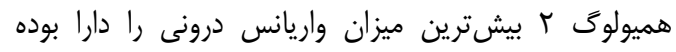

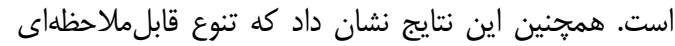


1. Abdoshahi, R A ., M. Omidi, R. Talei and B. Yazdi Samadi. 2009. Mapping QTLs controlling drought toler wheat (Triticum aestivum L.). Journal of Agricultural Research, 7: 527-539.

2. Aminian, R. 2010. Genomic analysis of yield, its components and traits associated with drought tolerance in wheat (Triticum aestivum L.). PhD Thesis, Faculty of Agriculture, Shahrekord University, Shahrekord, Iran. 240 pp (In Persian).

3. Aminian, R., S.H. Mohammady, S. Hoshmand and M. Khodombashi. 2011. Chromosomal analysis of Photosynthesis rate and stomatal conductance and their relationships with grain yield in wheat (Triticum aestivum L.) under water-stressed and well watered conditions. Acta physiologia plantarum, 33: 755-764.

4. Bartlett, M.S. 1937. Properties of sufficiency and statistical tests. Proceedings of the Royal Society of London Series AMathematical, Physical and Engineering Sciences. 160: 268-282.

5. Chandra, D., M. A. Islam and N.C.D. Barma. 2004. Variability and interrelationship of nine quantitative characters in F5 bulks of five wheat crosses. Pakistan Journal Biology Science, 6: 1040-1045.

6. Daud, H.M. and J.P. Gustafson. 1996. Molecular evidence for Triticum Speltoides as a B-genome progenitor of wheat (Triticum aestivum L.). Genome, 39: 543-548.

7. Ehdaie, B. and J.G. Waines. 1994. Genetic variation, heritability and path-analysis in landraces of bread wheat from southwestern Iran. Euphytica, 41: 183-190.

8. Farshadfar, E., B. Koszegi, T. Tischner and J. Sutka. 1995. Substitution analysis of drought tolerance in wheat (Triticum aestivum L.). Plant Breeding, 114: 542-548.

9. Farshadfar, E. and R. Mohammady. 2005. Genetic control analyze of drought tolerance in Chynne cultivar using substitution lines. Journal of Seed and Plant, 21(1): 93-108. (In Persian).

10. Giunta, F., R. Motzo and G. Pruneddu. 2007. Trends since 1900 in the yield potential of Italian-bred durum wheat cultivars. Europian Journal Agronomy, 27: 12-24.

11. Jiang, J., B. Friebe and B. S. Gill. 1994. Recent advances in alien gene transfer in wheat. Euphytica, 73: 199-212.

12. Khaled Fathy, M. 2004. The inheritance and molecular mapping of genes for post-anthesis drought tolerance in wheat. Electronic Ph.D document.

13.Khazaie, H., P. Monneveux, H. Shao and S. Mohammady, 2010. Variation for stomatal characteristics and water use efficiency among diploid, tetraploid and hexaplod Iranian wheat land races. Genetic Resources and Crop Evolution, 57: 307-314.

14. Kordenaeej, A. 2008. Mapping QTLs for yield and yield components under drought stress in bread wheat. Dissertation for a doctorate degree, University of Natural Resources and Applied Life Sciences (BOKU), Vienna, Austria, pp. 97.

15. Lelley, T., M. Stachel, H. Grausgruber and J. Vollmann .2000. Analysis of relationships between Aegilops tauschii and the D genome of wheat utilizing microsatellites. Genome, 43: 661-668.

16. Leopold, A.C. 1990. Coping with desiccation. In: Alscher, R. G. and J. R. Cumming (eds.) Stress response in plants: adaptation and acclimation mechanisms. 37-56 pp., Wiley-Liss, New York.

17. Levene, H. 1960. Robust testes for equality of variances. In: Olkin, I. (ed) Contributions to probability and statistics. 278-292 pp., Stanford University Press.

18.Maaroofi, A. 1988. Identification of chromosomes locations contributin to drought tolerance in wheat. M.Sc thesis, Faculty of Agriculture, Razi University, Iran. (In Persian).

19. Majer, P., L. Sass, T. Lelley, L. Cseuz, I. Vass, D. Dudits and J. Pauk. 2008. Testing drought tolerance of wheat by a complex stress diagnostic system installed in greenhouse. Acta Biologica Szegediensis, 52: 97-100.

20. Miller, T.E. 1987. Systematic and evolution. In: Lupton, F. G. H. (ed) Wheat Breeding: Its scientific basis. Chapman \& Hall. New York.

21. Mir, R., R.M. Zaman-Allah, N.Sreenivasulu, R. Trethowan and R.K. Varshney. 2012. Integrated genomiCS, physiology and breeding approaches for improving drought tolerance in crops. Theoretical and Applied Genetics, 125: 625-645.

22. Mohammadi, S.A. and B.M. Prasanna. 2003. Analysis of genetic diversity in cropplants- Salient statistical tools and considerations. Crop Science, 43: 1235-1248.

23. Mohammady, S. 2009. Chromosomal analysis for physiological traits related to drought resistance in bread wheat using monosomic lines. Shahrekord Universitv Press. 104 pd (In Persian).

24. Mohammadv. S. 2014. Variation and inheritance of carbon isotope discrimination in wheat (Triticum aestivum L.). Acta Physiologia plantarum, 36:2837-2844.

25.Mohammady, S. 2015. Variation for apical sterility among diploid, tetraploid and hexaploid Iranian wheats under meiotic stage water-stressed and well-watered conditions. Crop and Pasture Science, 66: 42-48.

26. Mohammady, S., R. Aminian, S. Hoshmand and M. Khodombashi. 2012. Genomic analysis of carbon isotope discrimination, photosynthesis rate, stomatal conductance, and grain yield in wheat (Triticum aestivum L.) under waterstressed conditions. Crop and Pasture Science, 63(6): 513-519.

27. Mohammady, S., Z. Heidari and S. Hooshmand. 2014. The determination of chromosomes involved in controlling epicuticular wax, water statues and stomatal characteristics using selected wheat substitution lines under water-stress conditions. Acta Physiology Plant, 36: 1325-1333.

28. Petersen, G., Seberg, Yde. Merete and K. Berthelsen. 2006. Phylogenetic relationships of Triticum and Aegilops and evidence for the origin of the A, B, and D genomes of common wheat (Triticum aestivum L.). Czech Journal Genetics. Plant Breeding, 41: 28-37.

29. Rosielle, A.A. and J. Hamblin. 1981. Theoretical aspects of selections for yield in stress and non-stress environments. Crop Science, 21: 943-946.

30. Steel, R. G. D. and J. H. Torrie. 1976. Introduction to statistics. McGrow-Hill: New York.

31. Zarkti, H., H. Ouabbou A. Hilali and S.M. Udupa. 2010. Detection of genetic diversity in Moroccan durum wheat accessions using agro-morphological traits and microsatellite markers. African Journal of Agricultural Research, 5(14): $1837-1844$

32. Zhang, L.Y., D.C. Liu , X.L. Guo, W.L. Yang, J.Z. Sun, D. Wang and A. Zhang . 2009. Distr ibution in genome of quantitativ e trait loci (QTL) for yield and yield-related traits in common wheat (Triticum aestivum L.). Theoretical and Applied Genetics, 119: 43-52. 


\title{
Evaluation of Intra Chromosome and Inter Hemeologous Variations for Grain Yield and Its Components under Non-Stress and Water-Stress Conditions using Wheat Chromosomal Substitution Lines
}

\author{
Mahnaz Alimohammadi ${ }^{1}$, Shahram Mohammady ${ }^{2}$, Behroz Shiran $^{3}$ and \\ Mohammad Rabiei ${ }^{4}$ \\ 1- Ph.D. Student, Shahrekord University (Corresponding author: alimohamadymahnaz@yahoo.com) \\ 2, 3 and 4- Associate Professor, Professor and Associate Professor, Shahrekord University \\ Received: May 8, 2016 \\ Accepted: June 13, 2016
}

\begin{abstract}
This study was conducted to evaluate intra-chromosome and inter hemeologous variations of grain yield and its components using two wheat substitution lines series including substitution lines of 'Timstein' into genetic background of 'Chinese Spring' and substitution lines of 'Red Egyptian' into genetic background of 'Chinese Spring' and their parents in a randomized complete block design with four replications under water-stress and non-stress conditions in a greenhouse. Analyses of variance indicated that significant differences exist within chromosomes and hemeologous groups for all studied traits under the both conditions. inter chromosome variations and inter hemeologous variations were significant at the level of 1 and 5 percent of probability, respectively. Homogeneity was performed using Bartlett's and Levene's tests for inter chromosomal and intra hemeologous variances. The results of these tests differed in water-stress conditions compared with normal ones. The results of homogeneity tests under non-stress conditions indicated that intra chromosomal homogeneity was significant for grain yield and weight of thousand grains. While under the water-stress conditions, these tests were significant for another two extra characters including harvest index and number of grains per spikelet. Similar to intra chromosome homogeneity, intra hemeologous hemogenety was also performed using Bartlett's and Levene's tests. Homologous groups in non-stress condition showed that the variances observed for 1000 seed weight were not homogeneous while variances observed for grain yield and harvest index were non homogeneous in the water-stress conditions. The hemeologous groups of wheat were statistically compared with each other for the characters indicated non homogenous variance using F-test. These comparisons indicated that 2 Homologous group was more effective in controlling variances observed for grain yield, harvest index and 1000 seed weight than other hemeologous groups. So it can be concluded that hemeologous grop 2 has the most important role in controlling the variance of yield and its components.
\end{abstract}

Keywords: Drought stress, inter-Chromosome variation, Intra Homologous chromosomes Variations, Substitution lines, Wheat 